\title{
Polar orbitopes
}

\author{
Leonardo Biliotti, Alessandro Ghigi and Peter Heinzner
}

We study polar orbitopes, i.e., convex hulls of orbits of a polar representation of a compact Lie group. They are given by representations of $K$ on $\mathfrak{p}$, where $K$ is a maximal compact subgroup of a real semisimple Lie group $G$ with Lie algebra $\mathfrak{g}=\mathfrak{k} \oplus \mathfrak{p}$. The face structure is studied by means of the gradient momentum map and it is shown that every face is exposed and is again a polar orbitope. Up to conjugation the faces are completely determined by the momentum polytope. There is a tight relation with parabolic subgroups: the set of extreme points of a face is the closed orbit of a parabolic subgroup of $G$ and for any parabolic subgroup the closed orbit is of this form.

1 Introduction $\quad 580$

2 Preliminaries $\quad 582$

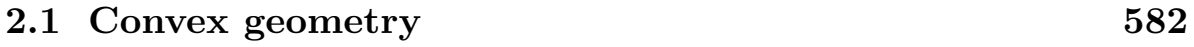

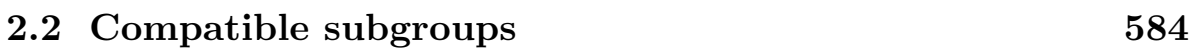

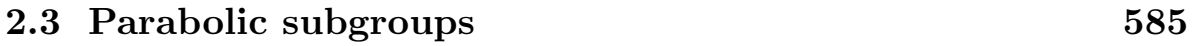

$\begin{array}{ll}2.4 \text { Gradient momentum map } & 587\end{array}$

$\begin{array}{ll}2.5 \text { Coadjoint orbits } & 589\end{array}$

$\begin{array}{lll}3 & \text { Face structure } & 590\end{array}$

$\begin{array}{lll}3.1 & \text { Faces as orbitopes } & 590\end{array}$

$\begin{array}{lll}3.2 & \text { All faces are exposed } & 594\end{array}$

$\begin{array}{lll}3.3 & \text { Faces and parabolic subgroups } & 598\end{array}$ 


\section{Introduction}

If $K$ is a compact group and $K \rightarrow \mathrm{Gl}(V)$ is a real representation, the convex hull of a $K$-orbit is called an orbitope [22]. If $V$ is provided with a $K$ invariant scalar product, the representation is said to be polar if there is a linear subspace $S \subset V$ that intersects perpendicularly all the orbits of $K$. An important class of examples is given by the adjoint representations of compact Lie groups. In [2] we studied the orbitopes of these actions. They are equivariantly isomorphic to Satake-Furstenberg compactifications of symmetric spaces of type $K^{\mathbb{C}} / K$. One homeomorphism has been described in algebraic terms in [17]. Another homeomorphism has been constructed in [1] (in the case of an integral orbit) using integration of the momentum map on a flag manifold. This geometric construction was developed by Bourguignon, $\mathrm{Li}$ and Yau in the case of $\mathbb{P}^{n}$.

In the present paper we study the orbitopes of a polar representation of a compact group. Let $G$ be a real connected semisimple Lie group and let $\mathfrak{g}=\mathfrak{k} \oplus \mathfrak{p}$ be a Cartan decomposition of its Lie algebra. Let $K$ be a maximal compact subgroup with Lie algebra $\mathfrak{k}$. Then the adjoint action of $K$ preserves $\mathfrak{p}$ and its restriction to $\mathfrak{p}$ is a polar representation. By a theorem of Dadok [5, Prop. 6] if $V$ is any polar representation of a group $K_{1}$, there is a semisimple Lie group $G$ such that $V$ can be identified with $\mathfrak{p}$ so that the orbits of $K_{1}$ coincide with the orbits of $\operatorname{Ad} K$ on $\mathfrak{p}$. Therefore to understand the orbitopes of polar representations it is sufficient to study the $K$-orbitopes on $\mathfrak{p}$.

The study of these orbitopes is also needed in order to generalize the results in [1] to general symmetric spaces and this is one of the motivations for our work.

Our set up is the following. Let $U$ be compact Lie group and let $U^{\mathbb{C}}$ be its complexification. A closed subgroup $G \subset U^{\mathbb{C}}$ is called compatible if $G=$ $K \cdot \exp \mathfrak{p}$ where $K:=G \cap U$ and $\mathfrak{p}:=\mathfrak{g} \cap i \mathfrak{u}$. It follows that $K$ is a maximal compact subgroup of $G$ and that $\mathfrak{g}=\mathfrak{k} \oplus \mathfrak{p}$. $K$ acts on $\mathfrak{g}$ by the adjoint action 
and $\mathfrak{p}$ is invariant. Therefore we get an action of $K$ on $\mathfrak{p}$. The objects that we wish to study are the orbits of this action and their convex hulls. It is easy to see that one can reduce to the case in which $U$ and $G$ are semisimple (see Section 3.2). If $\mathcal{O} \subset \mathfrak{p}$ is a $K$-orbit, we denote by $\widehat{\mathcal{O}}$ its convex hull. We will assume throughout the paper that $G$ is connected. It is a fundamental fact that the action of $K$ on $\mathcal{O}$ extends to an action of $G$, see, e.g., [12, Prop. 6]. If $\mathfrak{a} \subset \mathfrak{p}$ is a maximal subalgebra, then by Kostant convexity theorem [18], the orthogonal projection of $\mathcal{O}$ onto $\mathfrak{a}$ is a convex polytope $P$ given by the convex hull of a Weyl group orbit. In particular the Weyl group acts on the set $\mathscr{F}(P)$ of faces of $P$ and similarly $K$ acts on the set $\mathscr{F}(\widehat{\mathcal{O}})$ of faces of $\widehat{\mathcal{O}}$.

Our main result is the following.

Theorem 1.1. Let $P \subset \mathfrak{a}$ be the momentum polytope associated to $\mathcal{O}$. If $\sigma$ is a face of $P$ and $K^{\sigma^{\perp}}$ is the centralizer of the normal space $\sigma^{\perp} \subset \mathfrak{a}$, then $K^{\sigma^{\perp}} \cdot \sigma$ is a face of $\widehat{\mathcal{O}}$. Moreover the map $\sigma \mapsto K^{\sigma^{\perp}} \cdot \sigma$ induces a bijection between $\mathscr{F}(P) / W$ and $\mathscr{F}(\widehat{\mathcal{O}}) / K$.

The correspondence between $\mathscr{F}(\widehat{\mathcal{O}}) / K$ and $\mathscr{F}(P) / W$ holds for a general polar representation, see Remark 3.1 at p. 602. Applied to the case $G=U^{\mathbb{C}}$ this theorem gives the results proven in [2]. The setting of the present paper is more general than the one considered there. The pairs $(G, K)$ with $G$ compatible contain all Riemannian symmetric pairs of non-compact type, while the pairs $\left(U^{\mathbb{C}}, U\right)$ correspond to symmetric pairs of type IV $[13$, p. 516]. The particular cases $U=\mathrm{SU}(n), G=\mathrm{SL}(n, \mathbb{R})$ and $U=\mathrm{SO}(n), G=$ $\mathrm{SO}(n, \mathbb{C})$ have been considered in $[22]$. The case where $\mathcal{O}$ can be realized as the Shilov boundary of a Hermitian symmetric domain has been studied in [4, Prop. 2.1].

We outline the main steps of the proof.

Among the faces of a convex set are the exposed faces (see Section 2.1). In the case of $\widehat{\mathcal{O}}$ the study of these faces is equivalent to the understanding of the height functions on $\mathcal{O}$ (Section 3.1). This is a classical subject, going back to the paper [6] by Duistermaat et al. and to Heckman's thesis [8]. The results are very efficiently described in the language of the gradient momentum map (which is recalled in Section 2.4). The set of extreme points ext $F$ of an exposed face $F$ is connected and is an orbit of a centralizer $K^{\beta} \subset K$, where $\beta$ is an element of $\mathfrak{p}$ (Proposition 3.1). In general the group $K^{\beta}$ is not connected. An inductive argument shows that any face $F \subset \widehat{\mathcal{O}}$ (not necessarily exposed) is an orbitope of the centralizer $K^{\mathfrak{s}}$ of some subalgebra $\mathfrak{s} \subset \mathfrak{p}$ (Proposition 3.4). If $\mathfrak{a} \subset \mathfrak{p}$ is a maximal subalgebra containing $\mathfrak{s}$, we show that $F \cap \mathfrak{a}$ is a face of the momentum polytope and that $F \cap \mathfrak{a}$ determines 
$F$ (Proposition 3.6). Here we use in an essential way the Kostant convexity theorem.

An important conclusion is that all faces of $\widehat{\mathcal{O}}$ are exposed (Theorem 3.2). This answers Question 1 of [22] for polar orbitopes. Next recall that the $K$ action on $\mathcal{O}$ extends to an action of the group $G$ (see Section 2.5 below). We analyze the influence of the $G$-action on the geometry of the extreme points of the faces (Section 3.3). It turns out that there is a strong link between the parabolic subgroups of $G$ and the faces of $\widehat{\mathcal{O}}$. In 3.3 we show the following.

Theorem 1.2. The set $\{\operatorname{ext} F: F$ a non-empty face of $\widehat{\mathcal{O}}\}$ coincides with the set of all closed orbits of parabolic subgroups of $G$.

Using these results we finally set up the correspondence between the faces of $\widehat{\mathcal{O}}$ and the faces of $P$ and prove Theorem 1.1 (Section 3.4).

In the final section we briefly explain how the boundary of $\widehat{\mathcal{O}}$ is stratified by face type and how the Satake combinatorics can be used to describe the faces of the orbitope in terms of root data.

\section{Preliminaries}

\subsection{Convex geometry}

It is useful to recall a few definitions and results regarding convex sets (see, e.g., [24] and [2, Section 1]). Let $V$ be a real vector space with a scalar product $\langle$,$\rangle and let E \subset V$ be a compact convex subset. The relative interior of $E$, denoted relint $E$, is the interior of $E$ in its affine hull. A face $F$ of $E$ is a convex subset $F \subset E$ with the following property: if $x, y \in E$ and relint $[x, y] \cap F \neq \emptyset$, then $[x, y] \subset F$. The extreme points of $E$ are the points $x \in E$ such that $\{x\}$ is a face. Since $E$ is compact the faces are closed [24, p. 62]. A face distinct from $E$ and $\emptyset$ will be called a proper face. The support function of $E$ is the function $h_{E}: V \rightarrow \mathbb{R}, h_{E}(u)=\max _{x \in E}\langle x, u\rangle$. If $u \neq 0$, the hyperplane $H(E, u):=\left\{x \in E:\langle x, u\rangle=h_{E}(u)\right\}$ is called the supporting hyperplane of $E$ for $u$. The set

$$
F_{u}(E):=E \cap H(E, u)
$$

is a face and it is called the exposed face of $E$ defined by $u$. In general not all faces of a convex subset are exposed. A simple example is given by the convex hull of a closed disc and a point outside the disc: the resulting convex set is the union of the disc and a triangle. The two vertices of the triangle that lie on the boundary of the disc are non-exposed 0 -faces. 
Lemma 2.1 [2, Lemma 3]. If $F$ is a face of a convex set $E$, then $\operatorname{ext} F=$ $F \cap \operatorname{ext} E$.

Lemma 2.2. If $G$ is a compact group and $V$ is a representation space of $G$ define

$$
\rho: V \rightarrow V^{G} \quad \rho(v):=\int_{G} g x d g
$$

where dg denotes the Haar measure on $G$. Then $V=V^{G} \oplus \operatorname{ker} \rho$. If $x \in V$ and $x=x_{0}+x_{1}$ in this decomposition, then

(a) $G \cdot x=x_{0}+G \cdot x_{1}$;

(b) $\operatorname{conv}(G \cdot x)=x_{0}+\operatorname{conv}\left(G \cdot x_{1}\right)$;

(c) $x_{0}$ is the unique fixed point of $G$ contained in $\operatorname{conv}(G \cdot x)$;

(d) $x_{0} \in \operatorname{relint} \operatorname{conv}(G \cdot x)$.

Proof. That $V=V^{G} \oplus \operatorname{ker} \rho$ follows from the fact that $\operatorname{Im} \rho=V^{G}$ and $\rho^{2}=$ $\rho$. (a) and (b) are immediate. Since $x_{0}=\rho(x)$, it follows from the definition of $\rho$ that $x_{0} \in \operatorname{conv}(G \cdot x)$. If $y \in \operatorname{conv}(G \cdot x)$ is another fixed point, then $y_{0}=x_{0}$ and $y_{1} \in \operatorname{ker} \rho \cap V^{G}$. Hence $y_{1}=0$ and $y=x_{0}$. This proves (c). By Theorem 2.1 there is a unique face $F \subset \operatorname{conv}(G \cdot x)$ such that $x_{0} \in \operatorname{relint} F$. Since $\operatorname{conv}(G \cdot x)$ is $G$-invariant and $x_{0}$ is fixed by $G$, also $F$ is $G$-invariant, and hence also ext $F$. Since ext $F \subset \operatorname{ext}(\operatorname{conv}(G \cdot x))=G \cdot x$, it follows that ext $F=G \cdot x$ and hence that $F=\operatorname{conv}(G \cdot x)$.

Lemma 2.3 [2, Prop. 5]. If $F \subset E$ is an exposed face, the set $C_{F}:=\{u \in$ $\left.V: F=F_{u}(E)\right\}$ is a convex cone. If $G$ is a compact subgroup of $O(V)$ that preserves both $E$ and $F$, then $C_{F}$ contains a fixed point of $G$.

Theorem 2.1 [24, p. 62]. If $E$ is a compact convex set and $F_{1}, F_{2}$ are distinct faces of $E$, then relint $F_{1} \cap$ relint $F_{2}=\emptyset$. If $G$ is a non-empty convex subset of $E$ which is open in its affine hull, then $G \subset \operatorname{relint} F$ for some face $F$ of $E$. Therefore $E$ is the disjoint union of the relative interiors of its faces.

Lemma 2.4 [2, Lemma 7]. If $E$ is a compact convex set and $F \subsetneq E$ is a face, then $\operatorname{dim} F<\operatorname{dim} E$.

Lemma 2.5 [2, Lemma 8]. If $E$ is a compact convex set and $F \subset E$ is a face, then there is a chain of faces $F_{0}=F \subsetneq F_{1} \subsetneq \cdots \subsetneq F_{k}=E$ which is 
maximal, in the sense that for any $i$ there is no face of $E$ strictly contained between $F_{i-1}$ and $F_{i}$.

Lemma 2.6 [2, Lemma 9]. If $E$ is a convex subset of $\mathbb{R}^{n}, M \subset \mathbb{R}^{n}$ is an affine subspace and $F \subset E$ is a face, then $F \cap M$ is a face of $E \cap M$.

\subsection{Compatible subgroups}

(See $[10,11]$.$) If G$ is a Lie group with Lie algebra $\mathfrak{g}$ and $E, F \subset \mathfrak{g}$, we set

$$
\begin{aligned}
& E^{F}:=\{\eta \in E:[\eta, \xi]=0, \forall \xi \in F\}, \\
& G^{F}=\{g \in G: \operatorname{Ad} g(\xi)=\xi, \forall \xi \in F\} .
\end{aligned}
$$

If $F=\{\beta\}$ we write simply $E^{\beta}$ and $G^{\beta}$. Let $U$ be compact Lie group. Let $U^{\mathbb{C}}$ be its universal complexification which is a linear reductive complex algebraic group. We denote by $\theta$ both the conjugation map $\theta: \mathfrak{u}^{\mathbb{C}} \rightarrow \mathfrak{u}^{\mathbb{C}}$ and the corresponding group isomorphism $\theta: U^{\mathbb{C}} \rightarrow U^{\mathbb{C}}$. Let $f: U \times i \mathfrak{u} \rightarrow U^{\mathbb{C}}$ be the diffeomorphism $f(g, \xi)=g \exp \xi$. Let $G \subset U^{\mathbb{C}}$ be a closed subgroup. Set $K:=G \cap U$ and $\mathfrak{p}:=\mathfrak{g} \cap i \mathfrak{u}$. We say that $G$ is compatible if $f(K \times \mathfrak{p})=G$. The restriction of $f$ to $K \times \mathfrak{p}$ is then a diffeomorphism onto $G$. It follows that $K$ is a maximal compact subgroup of $G$ and that $\mathfrak{g}=\mathfrak{k} \oplus \mathfrak{p}$. Note that $G$ has finitely many connected components. Since $U$ can be embedded in $\operatorname{Gl}(N, \mathbb{C})$ for some $N$, and any such embedding induces a closed embedding of $U^{\mathbb{C}}$, any compatible subgroup is a closed linear group. Moreover $\mathfrak{g}$ is a real reductive Lie algebra, hence $\mathfrak{g}=\mathfrak{z}(\mathfrak{g}) \oplus[\mathfrak{g}, \mathfrak{g}]$. Denote by $G_{s s}$ the analytic subgroup tangent to $[\mathfrak{g}, \mathfrak{g}]$. Then $G_{s s}$ is closed and $G=Z(G)^{0} \cdot G_{s s}$ [16, p. 442].

Lemma 2.7. (a) If $G \subset U^{\mathbb{C}}$ is a compatible subgroup, and $H \subset G$ is closed and $\theta$-invariant, then $H$ is compatible if and only if $H$ has only finitely many connected components.

(b) If $G \subset U^{\mathbb{C}}$ is a connected compatible subgroup, then $G_{s s}$ is compatible.

(c) If $G \subset U^{\mathbb{C}}$ is a compatible subgroup, and $E \subset \mathfrak{p}$ is any subset, then $G^{E}$ is compatible.

Proof. This follows from the more general observation that a closed $\theta$ invariant subgroup $G \subset U^{\mathbb{C}}$ is compatible if and only if it has finitely many connected components. This is proven in Lemma 1.1 .3 in [19, p.14]. For the reader's convenience we recall the argument. If $G$ is compatible, then it 
retracts onto $K$, which is compact and therefore has finitely many connected components. Conversely assume that $G / G^{0}$ be finite. Since $G$ is closed, $f(K \times \mathfrak{p})$ is a closed subset of $G$. Since $G$ is $\theta$-invariant, $f(K \times \mathfrak{p})$ has the same dimension as $G$ and is therefore also open. Therefore it contains $G^{0}$ and is a union of connected components of $G$. Given $g \in G$ write $g=u \exp \xi$ with $u \in U$ and $\xi \in i \mathfrak{u}$. Then $g \theta\left(g^{-1}\right)=\exp (2 \operatorname{Ad}(u) \xi)$ and since $G / G^{0}$ is finite there is a natural number $N>0$ such that $\left(g \theta\left(g^{-1}\right)\right)^{N}=\exp (2 N \operatorname{Ad}(u) \xi) \in$ $G^{0}$. Hence $\operatorname{Ad}(u) \xi \in \mathfrak{p}, u=\exp (-\operatorname{Ad}(u) \xi) g \in G \cap U=K$ and $\xi \in \mathfrak{p}$. (b). Since $[\mathfrak{g}, \mathfrak{g}]$ is $\theta$-invariant and $G_{s s}$ is connected, $G_{s s}$ is $\theta$-invariant. Since it is also closed, it is compatible by (a). (c) see [16, Proposition 7.25 p. 452].

Let $\langle$,$\rangle be a fixed U$-invariant scalar product on $\mathfrak{u}$. We use it to identifiy $\mathfrak{u} \cong \mathfrak{u}^{*}$. We also denote by $\langle$,$\rangle the scalar product on i \mathfrak{u}$ such that multiplication by $i$ be an isometry of $\mathfrak{u}$ onto $i \mathfrak{u}$. One can define an $\mathbb{R}$-bilinear form $B$ on $\mathfrak{u}^{\mathbb{C}}$ by imposing $B(\mathfrak{u}, i \mathfrak{u})=0, B=-\langle$,$\rangle on \mathfrak{u}$ and $B=\langle$,$\rangle on i \mathfrak{u}$. Then $B$ is $\operatorname{Ad} U^{\mathbb{C}}$-invariant and non-degenerate.

\subsection{Parabolic subgroups}

(See, e.g., [3, p. 28ff], [16].) If $G \subset U^{\mathbb{C}}$ is compatible, $\mathfrak{g}=\mathfrak{k} \oplus \mathfrak{p}$ is reductive. A subalgebra $\mathfrak{q} \subset \mathfrak{g}$ is parabolic if $\mathfrak{q}^{\mathbb{C}}$ is a parabolic subalgebra of $\mathfrak{g}^{\mathbb{C}}$. One way to describe the parabolic subalgebras of $\mathfrak{g}$ is by means of restricted roots. If $\mathfrak{a} \subset \mathfrak{p}$ is a maximal subalgebra, let $\Delta(\mathfrak{g}, \mathfrak{a})$ be the (restricted) roots of $\mathfrak{g}$ with respect to $\mathfrak{a}$, let $\mathfrak{g}_{\lambda}$ denote the root space corresponding to $\lambda$ and let $\mathfrak{g}_{0}=\mathfrak{m} \oplus \mathfrak{a}$, where $\mathfrak{m}=\mathfrak{z}_{\mathfrak{k}}(\mathfrak{a})$. Let $\Pi \subset \Delta(\mathfrak{g}, \mathfrak{a})$ be a base and let $\Delta_{+}$be the set of positive roots. If $I \subset \Pi$ set $\Delta_{I}:=\operatorname{span}(I) \cap \Delta$. Then

$$
\mathfrak{q}_{I}:=\mathfrak{g}_{0} \oplus \bigoplus_{\lambda \in \Delta_{I} \cup \Delta_{+}} \mathfrak{g}_{\lambda}
$$

is a parabolic subalgebra. Conversely, if $\mathfrak{q} \subset \mathfrak{g}$ is a parabolic subalgebra, then there are a maximal subalgebra $\mathfrak{a} \subset \mathfrak{p}$ contained in $\mathfrak{q}$, a base $\Pi \subset \Delta(\mathfrak{g}, \mathfrak{a})$ and a subset $I \subset \Pi$ such that $\mathfrak{q}=\mathfrak{q}_{I}$. We can further introduce

$$
\begin{aligned}
\mathfrak{a}_{I} & :=\bigcap_{\lambda \in I} \operatorname{ker} \lambda \quad \mathfrak{a}^{I}:=\mathfrak{a}_{I}^{\perp}, \\
\mathfrak{n}_{I} & =\bigoplus_{\lambda \in \Delta_{+}-\Delta_{I}} \mathfrak{g}_{\lambda} \quad \mathfrak{m}_{I}:=\mathfrak{m} \oplus \mathfrak{a}^{I} \oplus \bigoplus_{\lambda \in \Delta_{I}} \mathfrak{g}_{\lambda} .
\end{aligned}
$$

Then $\mathfrak{q}_{I}=\mathfrak{m}_{I} \oplus \mathfrak{a}_{I} \oplus \mathfrak{n}_{I}$. Since $\theta \mathfrak{g}_{\lambda}=\mathfrak{g}_{-\lambda}$, it follows that $\mathfrak{q}_{I} \cap \theta \mathfrak{q}_{I}=\mathfrak{a}_{I} \oplus \mathfrak{m}_{I}$. This latter Lie algebra coincides with the centralizer of $\mathfrak{a}_{I}$ in $\mathfrak{g}$. It is a Levi 
factor of $\mathfrak{q}_{I}$ and

$$
\mathfrak{a}_{I}=\mathfrak{z}\left(\mathfrak{q}_{I} \cap \theta \mathfrak{q}_{I}\right) \cap \mathfrak{p} .
$$

Another way to describe parabolic subalgebras of $\mathfrak{g}$ is the following. If $\beta \in \mathfrak{p}$, the endomorphism $\operatorname{ad} \beta \in$ End $\mathfrak{g}$ is diagonalizable over $\mathbb{R}$. Denote by $V_{\lambda}(\operatorname{ad} \beta)$ the eigenspace of $\operatorname{ad} \beta$ corresponding to the eigenvalue $\lambda$. Set

$$
\mathfrak{g}^{\beta+}:=\bigoplus_{\lambda \geq 0} V_{\lambda}(\operatorname{ad} \beta) .
$$

Lemma 2.8. For any $\beta$ in $\mathfrak{p}, \mathfrak{g}^{\beta+}$ is a parabolic subalgebra of $\mathfrak{g}$. If $\mathfrak{q} \subset \mathfrak{g}$ is a parabolic subalgebra, there is some vector $\beta \in \mathfrak{p}$ such that $\mathfrak{q}=\mathfrak{g}^{\beta+}$. The set of all such vectors is an open convex cone in $\mathfrak{z}(\mathfrak{q} \cap \theta \mathfrak{q}) \cap \mathfrak{p}$.

Proof. Given $\beta$ choose a maximal subalgebra $\mathfrak{a}$ containing $\beta$ and a base $\Pi \subset$ $\Delta(\mathfrak{g}, \mathfrak{a})$ such that $\beta$ lies in the closure of the positive Weyl chamber. Then $\mathfrak{g}^{\beta+}=\mathfrak{q}_{I}$ with $I:=\{\lambda \in \Pi: \lambda(\beta)=0\}$. This proves the first assertion. To prove the second fix a parabolic subalgebra $\mathfrak{q}$ and set $\Omega:=\left\{\beta \in \mathfrak{p}: \mathfrak{g}^{\beta+}=\mathfrak{q}\right\}$. Let $\mathfrak{a}$ be any maximal subalgebra of $\mathfrak{p}$ contained in $\mathfrak{q}$. Then $\mathfrak{q}=\mathfrak{q}_{I}$ for some $I \subset \Pi$ and

$$
\Omega \cap \mathfrak{a}=\left\{\beta \in \mathfrak{a}_{I}: \lambda(\beta)>0 \quad \text { for } \lambda \in \Pi-I\right\} .
$$

Thus $\Omega \cap \mathfrak{a}$ is a non-empty open convex cone in $\mathfrak{a}_{I}$. Therefore $\Omega \neq \emptyset$, which proves the second assertion. By $(2.4) \mathfrak{a}_{I}=\mathfrak{z}(\mathfrak{q} \cap \theta \mathfrak{q}) \cap \mathfrak{p}$, so $\Omega \cap \mathfrak{a}$ is an open convex cone in $\mathfrak{z}(\mathfrak{q} \cap \theta \mathfrak{q}) \cap \mathfrak{p}$. Moreover for any $\beta \in \Omega, \mathfrak{a} \subset \mathfrak{q} \cap \theta(\mathfrak{q})=\mathfrak{g}^{\beta}$. Thus $[\beta, \mathfrak{a}]=0$, hence $\beta \in \mathfrak{a}$. So $\Omega \subset \mathfrak{a}$, i.e., $\Omega=\Omega \cap \mathfrak{a}$.

A parabolic subgroup of $G$ is a subgroup of the form $Q=N_{G}(\mathfrak{q})$ where $\mathfrak{q}$ is a parabolic subalgebra of $\mathfrak{g}$. Equivalently, a parabolic subgroup of $G$ is a subgroup of the form $P \cap G$, where $P$ is parabolic subgroup of $G^{\mathbb{C}}$ and $\mathfrak{p}$ is the complexification of a subspace $\mathfrak{q} \subset \mathfrak{g}$. If $\beta \in \mathfrak{p}$ set

$$
\begin{aligned}
G^{\beta+} & :=\left\{g \in G: \lim _{t \rightarrow-\infty} \exp (t \beta) g \exp (-t \beta) \text { exists }\right\} \\
R^{\beta+} & :=\left\{g \in G: \lim _{t \rightarrow-\infty} \exp (t \beta) g \exp (-t \beta)=e\right\} \\
\mathfrak{r}^{\beta+} & :=\bigoplus_{\lambda>0} V_{\lambda}(\operatorname{ad} \beta) .
\end{aligned}
$$

Note that $\mathfrak{g}^{\beta+}=\mathfrak{g}^{\beta} \oplus \mathfrak{r}^{\beta+}$. 
Lemma 2.9. $G^{\beta+}$ is a parabolic subgroup of $G$ with Lie algebra $\mathfrak{g}^{\beta+}$. Every parabolic subgroup of $G$ equals $G^{\beta+}$ for some $\beta \in \mathfrak{p} . R^{\beta+}$ is the unipotent radical of $G^{\beta+}$ and $G^{\beta}$ is a Levi factor.

Proof. It is easy to check that $G^{\beta+}$ is a subgroup and that $G^{\beta+}=\left(G^{\mathbb{C}}\right)^{\beta+} \cap$ $G$. Therefore it is enough to prove that $\left(G^{\mathbb{C}}\right)^{\beta+}$ is parabolic. In other words we can assume that $G$ is a complex reductive group. If $X \in \mathfrak{g}$, then

$$
\exp (t \beta) \exp X \exp (-t \beta)=\exp (\operatorname{Ad}(\exp (t \beta)) \cdot X)=\exp \left(e^{\operatorname{tad} \beta} \cdot X\right)
$$

where $e^{\operatorname{tad} \beta}$ denotes the exponential in $\operatorname{End}(\mathfrak{g})$. Let $\Omega \subset \mathfrak{g}$ be a neighborhood of 0 such that exp is a diffeomorphism on $\Omega$. If $X \in \Omega$, then $\exp X \in R^{\beta+}$ if and only if $\lim _{t \rightarrow-\infty} e^{\operatorname{tad} \beta} \cdot X=0$ if and only if $X \in \mathfrak{r}^{\beta+}$. This shows that $R^{\beta+}$ is locally closed, hence closed [13, Prop. 2.11 p. 119]. Next observe that if $g \in G^{\beta+}$, and

$$
a:=\lim _{t \rightarrow-\infty} \exp (t \beta) g \exp (-t \beta),
$$

then $a \in G^{\beta} \subset G^{\beta+}$ and $a^{-1} g \in R^{\beta+}$. Therefore $G^{\beta+}$ is the product of the two closed subgroups $G^{\beta}$ and $R^{\beta+}$ and $G^{\beta} \cap R^{\beta+}=\{e\}$. It follows that $G^{\beta+}$ is a Lie subgroup of $G$ tangent to $\mathfrak{g}^{\beta+}$. Since we are now assuming that $G$ is complex, then it is well-known that $G^{\beta+}$ is closed and parabolic since its Lie algebra is parabolic.

\subsection{Gradient momentum map}

Let $(Z, \omega)$ be a Kähler manifold. Assume that $U^{\mathbb{C}}$ acts holomorphically on $Z$, that $U$ preserves $\omega$ and that there is a momentum map $\mu: Z \rightarrow \mathfrak{u}$. If $\xi \in \mathfrak{u}$ we denote by $\xi_{Z}$ the induced vector field on $Z$ and we let $\mu^{\xi} \in C^{\infty}(Z)$ be the function $\mu^{\xi}(z):=\langle\mu(z), \xi\rangle$. That $\mu$ is the momentum map means that it is $U$-equivariant and that $d \mu^{\xi}=i_{\xi_{Z}} \omega$.

Let $G \subset U^{\mathbb{C}}$ be compatible. If $z \in Z$, let $\mu_{\mathfrak{p}}(z) \in \mathfrak{p}$ denote $-i$ times the component of $\mu(z)$ in the direction of $i \mathfrak{p}$. In other words we require that $\left\langle\mu_{\mathfrak{p}}(z), \beta\right\rangle=-\langle\mu(z), i \beta\rangle$ for any $\beta \in \mathfrak{p}$. (Recall that multiplication by $i$ is an isometry of $\mathfrak{u}$ onto $i \mathfrak{u}$.) We have thus defined the gradient momentum map

$$
\mu_{\mathfrak{p}}: Z \rightarrow \mathfrak{p}
$$

Let $\mu_{\mathfrak{p}}^{\beta} \in C^{\infty}(Z)$ be the function $\mu_{\mathfrak{p}}^{\beta}(z)=\left\langle\mu_{\mathfrak{p}}(z), \beta\right\rangle=\mu^{-i \beta}(z)$. Let $($, ) be the Kähler metric associated to $\omega$, i.e., $(v, w)=\omega(v, J w)$. Then $\beta_{Z}$ is the gradient of $\mu_{\mathfrak{p}}^{\beta}$. If $X \subset Z$ is a locally closed $G$-invariant submanifold, then 
$\beta_{X}$ is the gradient of $\left.\mu_{\mathfrak{p}}^{\beta}\right|_{X}$ with respect to the induced Riemannian structure on $X$.

Theorem 2.2 (Slice Theorem [10, Thm. 3.1]). If $x \in X$ and $\mu_{\mathfrak{p}}(x)=0$, there are a $G_{x}$-invariant decomposition $T_{x} X=\mathfrak{g} \cdot x \oplus W$, open $G_{x}$-invariant subsets $S \subset W, \Omega \subset X$ and a $G$-equivariant diffeomorphism $\Psi: G \times{ }^{G_{x}} S \rightarrow$ $\Omega$, such that $0 \in S, x \in \Omega$ and $\Psi([e, 0])=x$.

Here $G \times{ }^{G_{x}} S$ denotes the associated bundle with principal bundle $G \rightarrow$ $G / G_{x}$.

Corollary 2.1. If $x \in X$ and $\mu_{\mathfrak{p}}(x)=\beta$, there are a $G^{\beta}$-invariant decomposition $T_{x} X=\mathfrak{g}^{\beta} \cdot x \oplus W$, open $G^{\beta}$-invariant subsets $S \subset W, \Omega \subset X$ and a $G^{\beta}$-equivariant diffeomorphism $\Psi: G^{\beta} \times^{G_{x}} S \rightarrow \Omega$, such that $0 \in S, x \in \Omega$ and $\Psi([e, 0])=x$.

This follows applying the previous theorem to the action of $G^{\beta}$ with the momentum map $\widehat{\mu_{\mathfrak{u}^{\beta}}}:=\mu_{\mathfrak{u}^{\beta}}-\mathrm{i} \beta$, where $\mu_{\mathfrak{u}^{\beta}}$ denotes the projection of $\mu$ onto $\mu_{\mathfrak{u}^{\beta}}$. See $[10$, p. 169] for more details.

If $\beta \in \mathfrak{p}$, then $\beta_{X}$ is a vector field on $X$, i.e., a section of $T X$. For $x \in X$, the differential is a map $T_{x} X \rightarrow T_{\beta_{X}(x)}(T X)$. If $\beta_{X}(x)=0$, there is a canonical splitting $T_{\beta_{X}(x)}(T X)=T_{x} X \oplus T_{x} X$. Accordingly $d \beta_{X}(x)$ splits into a horizontal and a vertical part. The horizontal part is the identity map. We denote the vertical part by $d \beta_{X}(x)$. It belongs to $\operatorname{End}\left(T_{x} X\right)$. Let $\left\{\varphi_{t}=\exp (t \beta)\right\}$ be the flow of $\beta_{X}$. There is a corresponding flow on $T X$. Since $\varphi_{t}(x)=x$, the flow on $T X$ preserves $T_{x} X$ and there it is given by $d \varphi_{t}(x) \in \operatorname{Gl}\left(T_{x} X\right)$. Thus we get a linear $\mathbb{R}$-action on $T_{x} X$ with infinitesimal generator $d \beta_{X}(x)$.

Corollary 2.2. If $\beta \in \mathfrak{p}$ and $x \in X$ is a critical point of $\mu_{\mathfrak{p}}^{\beta}$, then there are open invariant neighborhoods $S \subset T_{x} X$ and $\Omega \subset X$ and an $\mathbb{R}$-equivariant diffeomorphism $\Psi: S \rightarrow \Omega$, such that $0 \in S, x \in \Omega, \Psi(0)=x$. (Here $t \in \mathbb{R}$ acts as $d \varphi_{t}(x)$ on $S$ and as $\varphi_{t}$ on $\Omega$.)

Proof. The subgroup $H:=\exp (\mathbb{R} \beta)$ is compatible. It is enough to apply the previous corollary to the $H$-action at $x$.

Assume now that $\beta \in \mathfrak{p}$ and that $x \in \operatorname{Crit}\left(\mu_{\mathfrak{p}}^{\beta}\right)$. Let $D^{2} \mu_{\mathfrak{p}}^{\beta}(x)$ denote the Hessian, which is a symmetric operator on $T_{x} X$ such that

$$
\left(D^{2} \mu_{\mathfrak{p}}^{\beta}(x) v, v\right)=\frac{d^{2}}{d t^{2}}\left(\mu_{\mathfrak{p}}^{\beta} \circ \gamma\right)(0)
$$


where $\gamma$ is a smooth curve, $\gamma(0)=x$ and $\dot{\gamma}(0)=v$. Denote by $V_{-}$(respectively $V_{+}$) the sum of the eigenspaces of the Hessian of $\mu_{\mathfrak{p}}^{\beta}$ corresponding to negative (resp. positive) eigenvalues. Denote by $V_{0}$ the kernel. Since the Hessian is symmetric we get an orthogonal decomposition

$$
T_{x} X=V_{-} \oplus V_{0} \oplus V_{+}
$$

Let $\alpha: G \rightarrow X$ be the orbit map: $\alpha(g):=g x$. The differential $d \alpha_{e}$ is the map $\xi \mapsto \xi_{X}(x)$.

Proposition 2.1. If $\beta \in \mathfrak{p}$ and $x \in \operatorname{Crit}\left(\mu_{\mathfrak{p}}^{\beta}\right)$ then

$$
D^{2} \mu_{\mathfrak{p}}^{\beta}(x)=d \beta_{X}(x)
$$

Moreover, $d \alpha_{e}\left(\mathfrak{r}^{\beta \pm}\right) \subset V_{ \pm}$and $d \alpha_{e}\left(\mathfrak{g}^{\beta}\right) \subset V_{0}$. If $X$ is $G$-homogeneous these are equalities.

Proof. The first statement is proved in [10, Prop. 2.5]. Denote by $\rho: G_{x} \rightarrow$ $T_{x} X$ the isotropy representation: $\rho(g)=d g_{x}$. Observe that $\alpha$ is $G_{x^{-}}$ equivariant where $G_{x}$ acts on $G$ by conjugation, hence $d \alpha_{e}$ is $G_{x}$-equivariant, where $G_{x}$ acts on $\mathfrak{g}$ by the adjoint representation and on $T_{x} X$ by the isotropy representation. Since $\beta_{X}(x)=0, \exp (t \beta) \in G_{x}$ for any $t$ and $d \alpha_{e}$ is $\mathbb{R}$-equivariant. Therefore it interchanges the infinitesimal generators of the $\mathbb{R}$-actions, i.e., $d \alpha_{e} \circ \operatorname{ad} \beta=d \beta_{X}=D^{2} \mu_{\mathfrak{p}}^{\beta}(x)$. The required inclusions follow. If $G$ acts transitively on $X$ we must have $T_{x} X=d \alpha_{e}(\mathfrak{g})$. Hence the three inclusions must be equalities.

Corollary 2.3. For every $\beta \in \mathfrak{p}, \mu_{\mathfrak{p}}^{\beta}$ is a Morse-Bott function.

Proof. Let $X^{\beta}:=\left\{x \in X: \beta_{X}(x)=0\right\}$. Corollary 2.2 implies that $X^{\beta}$ is a smooth submanifold. Since $T_{x} X^{\beta}=V_{0}$ for $x \in X^{\beta}$, the first statement of Proposition 2.1 shows that the Hessian is non-degenerate in the normal directions.

\subsection{Coadjoint orbits}

Let $U$ be a compact connected semisimple Lie group. Fix a scalar product $\langle$,$\rangle on \mathfrak{u}$ and identify $\mathfrak{u}^{*} \cong \mathfrak{u}$. Let $z \in \mathfrak{u}$ and let $Z:=U \cdot z$ (adjoint action). $Z$ is a (co)adjoint orbit, hence it is provided with the Kostant-Kirillov-Souriau 
symplectic form which is defined by

$$
\omega_{z}\left(v_{Z}, w_{Z}\right):=\langle x,[v, w]\rangle \quad v, w \in \mathfrak{k} .
$$

(See, e.g., [15, p. 5].) The inclusion $Z \hookrightarrow \mathfrak{u}$ is the momentum map for the $U$-action on $Z$. Set $Q:=\left(U^{\mathbb{C}}\right)^{z+}$. Then $Q$ is a parabolic subgroup of $U^{\mathbb{C}}$ and $T_{z} Z \cong \mathfrak{u}^{\mathbb{C}} / \mathfrak{q}$. This endows $Z$ with an invariant complex structure $J$ such that $\omega$ is an invariant Kähler form. Such a structure is in fact unique. The action of $U$ on $Z$ extends to a holomorphic action of $U^{\mathbb{C}}$.

To study $K$-orbits on $\mathfrak{p}$ it is convenient to identify $\mathfrak{p}$ with $i \mathfrak{p}$ by multiplying by $i$. A $K$-orbit $\mathcal{O}=K \cdot x \subset \mathfrak{p}$ is mapped to $K \cdot i x \subset Z:=U \cdot i x$. Since $G \subset U^{\mathbb{C}}, G$ acts on $Z$ and we have $G \cdot i x=K \cdot i x$, see [11, Lemma 5] for the case $G^{\mathbb{C}}=U^{\mathbb{C}}$ and [12, Prop. 6] for the general case. Therefore the data $G, K, U, Z, X$ are like in the previous setting. And identifying $\mathcal{O} \cong K \cdot i x$, the gradient momentum becomes the inclusion $\mathcal{O} \subset \mathfrak{p}$.

\section{Face structure}

\subsection{Faces as orbitopes}

Let $U$ be a compact Lie group and let $G \subset U^{\mathbb{C}}$ be a compatible connected subgroup.

Definition 3.1. An orbitope of $G$ is the convex envelope of a $K$-orbit in $\mathfrak{p}$. If $\mathcal{O} \subset \mathfrak{p}$ is the $K$-orbit in $\mathfrak{p}, \widehat{\mathcal{O}}$ denotes the corresponding orbitope.

Lemma 3.1. We have ext $\widehat{\mathcal{O}}=\mathcal{O}$ and $\operatorname{ext} F=F \cap \mathcal{O}$ for any face $F$ of $\widehat{\mathcal{O}}$. Proof. This fact is common to all orbitopes, see [22, Prop. 2.2] or [2, Lemma 14].

We start the analysis of the structure of the faces of $\widehat{\mathcal{O}}$ by considering the exposed faces. At the end of Section 3.2 we will prove that in fact all faces of $\widehat{\mathcal{O}}$ are exposed. Let $\beta$ be a non-zero vector in $\mathfrak{p}$. Since $\mu_{\mathfrak{p}}$ is the inclusion $\mathcal{O} \hookrightarrow \mathfrak{p}$, the function $\mu_{\mathfrak{p}}^{\beta}$ is $\mu_{\mathfrak{p}}^{\beta}(x):=\langle x, \beta\rangle$. Set

$$
\operatorname{Max}(\beta):=\left\{x \in \mathcal{O}: \mu_{\mathfrak{p}}^{\beta}(x)=\max _{\mathcal{O}} \mu_{\mathfrak{p}}^{\beta}\right\}
$$

The main result about this set is the following.

Proposition 3.1. The set $\operatorname{Max}(\beta)$ is a connected $K^{\beta}$-orbit. In particular it is a $\left(K^{\beta}\right)^{0}$-orbit. 
This theorem goes back to $[6,8]$. Since it is basic we repeat the proof in our context. If $\mathfrak{a} \subset \mathfrak{p}$ is a maximal subalgebra, we denote by $W=W(\mathfrak{k}, \mathfrak{a})$ the Weyl group of $\mathfrak{a}$ in $K$.

Lemma 3.2. Let $\mathfrak{g}$ be a real semisimple Lie algebra with Cartan decomposition $\mathfrak{g}=\mathfrak{k} \oplus \mathfrak{p}$ and let $\mathfrak{a} \subset \mathfrak{p}$ be a maximal subalgebra. If $x, y \in \mathfrak{a}$ then there is a Weyl chamber $C$ such that $\bar{C}$ contains both $x$ and $y$ if and only if $\lambda(x) \lambda(y) \geq 0$ for every restricted root $\lambda$.

Proof (see [8, p. 11]). A Weyl chamber is a connected component of the set where all roots are non-zero. Given such a component $C$, let $\Delta_{+}$be the set of roots that are positive on $C$. Then $\Delta=\Delta_{+} \sqcup\left(-\Delta_{+}\right)$. From this follows the "only if" part. To prove the "if" part we can assume that $x$ and $y$ are different. Let $z:=(x+y) / 2$ and let $C$ be a Weyl chamber with $z \in \bar{C}$. By assumption, no root changes its sign on the segment $[x, y]$. Therefore $\lambda(z)>$ 0 implies that $\lambda(x) \geq 0$ and $\lambda(y) \geq 0$. If $\lambda(z)=0$, then $\lambda(x)=\lambda(y)=0$. Therefore $x$ and $y$ belong to $\bar{C}$. We thank the referee for pointing out this short argument.

Lemma 3.3. Let $C \subset \mathfrak{a}$ be a Weyl chamber and let $x, y \in \bar{C}$. If $x^{\prime} \in W \cdot x$, then there is a Weyl chamber $C^{\prime}$ such that $x^{\prime}, y \in \overline{C^{\prime}}$ if and only if there is $w \in W$ such that $w \cdot x=x^{\prime}$ and $w \cdot y=y$.

Proof. The "if" part follows from the definition of a Weyl chamber. Assume the existence of a Weyl chamber $C^{\prime}$ such that $x^{\prime}, y \in \overline{C^{\prime}}$. Then $x^{\prime}=\sigma x$ for some $\sigma \in W$. Let $w \in W$ be such that $w(C)=C^{\prime}$. The points $w^{-1} x^{\prime}=$ $w^{-1} \sigma x \in$ and $x$ belong to $\bar{C}$ and to the same Weyl orbit. Hence $w^{-1} x^{\prime}=$ $w^{-1} \sigma x=x\left[14\right.$, p. 52], i.e., $x^{\prime}=w x$. Also $w^{-1} y$ and $y$ belong to $\bar{C}$. Hence also $w y=y$. This concludes the proof.

Proposition 3.2. Let $G$ be a real connected semisimple Lie group. Let $\beta \in \mathfrak{p}$.

(a) If $\mathfrak{a} \subset \mathfrak{p}^{\beta}$ is a maximal subalgebra, then

$$
\mathfrak{p}^{\beta}=\bigcup_{k \in\left(K^{\beta}\right)^{0}} \operatorname{Ad}(k) \mathfrak{a} .
$$

(b) Let $W^{\beta}:=\{w \in W: w \beta=\beta\}$. Then for any $w \in W^{\beta}$ there is a $k \in$ $\left(K^{\beta}\right)^{0}$ such that $\operatorname{Ad}(k) \mathfrak{a}=\mathfrak{a}$ and $\operatorname{Ad}(k) x=w \cdot x$ for every $x \in \mathfrak{a}$.

For a proof see for example [16, p. 378-379, 383, 455-457]). 
Lemma 3.4. $\operatorname{Crit}\left(\mu_{\mathfrak{p}}^{\beta}\right)=\mathcal{O} \cap \mathfrak{p}^{\beta}$.

Proof. Let $Z$ be the $U$-orbit containing $\mathcal{O}$ as in Section 2.5. As observed in Section $2.4 \operatorname{grad} \mu_{\mathfrak{p}}^{\beta}=\left.\beta_{Z}\right|_{\mathcal{O}}$. So the set of critical points of $\mu_{\mathfrak{p}}^{\beta}$ on $\mathcal{O}$ is the set of zeros of $\beta_{Z}$ on $Z$ intersected with $\mathcal{O}$. Since $(i \beta)_{Z}(x)=[i \beta, x]$, we have $\operatorname{Crit}\left(\mu_{\mathfrak{p}}^{\beta}\right)=\mathcal{O} \cap \mathfrak{p}^{\beta}$.

Lemma 3.5. Let $G$ be semisimple. Fix $x \in \operatorname{Crit}\left(\mu_{\mathfrak{p}}^{\beta}\right)$. Let $\mathfrak{a} \subset \mathfrak{p}$ be a maximal subalgebra containing both $x$ and $\beta$. Then

$$
\operatorname{Crit}\left(\mu_{\mathfrak{p}}^{\beta}\right)=\bigcup_{w \in W}\left(K^{\beta}\right)^{0} \cdot w \cdot x=\left(K^{\beta}\right)^{0} \cdot N_{K}(\mathfrak{a}) \cdot x,
$$

where $W=W(\mathfrak{k}, \mathfrak{a})$ is the Weyl group.

Proof. Let $z \in \operatorname{Crit}\left(\mu_{\mathfrak{p}}^{\beta}\right)=\mathcal{O} \cap \mathfrak{p}^{\beta}$. By Proposition 3.2 there is $k \in\left(K^{\beta}\right)^{0}$ such that $k \cdot z \in \mathfrak{a}$. But $k \cdot z \in \mathcal{O}$ and $\mathcal{O} \cap \mathfrak{a}=W \cdot x$.

Proposition 3.3. Let $G$ be semisimple. Assume that $x \in \mathcal{O} \cap \mathfrak{a}$ and $\beta \in \mathfrak{a}$. Then $x$ is a local maximum of $\mu_{\mathfrak{p}}^{\beta}$ if and only if there exists a Weyl chamber $C \subset \mathfrak{a}$ such that $x, \beta \in \bar{C}$.

Proof. Let $\Delta$ be the set of restricted roots of $(\mathfrak{g}, \mathfrak{a})$ and let $\xi=\xi_{0}+\sum_{\lambda \in \Delta} \xi_{\lambda}$ with $\xi_{\lambda} \in \mathfrak{g}_{\lambda}$. Fix a set of positive roots $\Delta_{+}$such that $\lambda(x) \geq 0$ for every $\lambda \in \Delta_{+}$. We have

$$
\mathfrak{k}=\mathfrak{z} \mathfrak{k}(\mathfrak{a}) \oplus \bigoplus_{\lambda \in \Delta_{+}}\left(\mathfrak{g}_{\lambda} \oplus \mathfrak{g}_{-\lambda}\right) \cap \mathfrak{k} .
$$

(See, e.g., [16, p. 370].) Since $T_{x} \mathcal{O}=\mathfrak{k} \cdot x=[\mathfrak{k}, x]$ and $\left[x, \mathfrak{g}_{\lambda}\right]=\mathfrak{g}_{\lambda}$ if $\lambda(x) \neq 0$ and $\left[x, \mathfrak{g}_{\lambda}\right]=0$ otherwise, we have

$$
T_{x} \mathcal{O}=\bigoplus_{\lambda(x)>0}\left(\mathfrak{g}_{\lambda} \oplus \mathfrak{g}_{-\lambda}\right) \cap \mathfrak{p} .
$$

If $w \in T_{x} \mathcal{O}$, choose $\xi \in \mathfrak{k}$ such that $w=\xi_{\mathcal{O}}(x)=[\xi, x]$ and set $\gamma(t):=$ $\operatorname{Ad}(\exp (t \xi)) \cdot x$. Then $\gamma(0)=x, \dot{\gamma}(t)=[\xi, \gamma(t)], \ddot{\gamma}(0)=[\xi,[\xi, x]]$ and

$$
D^{2} \mu_{\mathfrak{p}}^{\beta}(x)(w, w)=\left.\frac{d}{d t}\right|_{t=0} \mu_{\mathfrak{p}}^{\beta}(\gamma(t))=\langle\ddot{\gamma}(0), \beta\rangle=-\langle[\xi, x],[\xi, \beta]\rangle .
$$

We can assume that $\xi=\sum_{\lambda(x)>0} \xi_{\lambda}$ with $\xi_{\lambda} \in \mathfrak{g}_{\lambda}$. This determines $\xi$ uniquely. Then

$$
[x, \xi]=\sum_{\lambda(x)>0} \lambda(x) z_{\lambda}
$$


where $z_{\lambda}=\xi_{\lambda}-\xi_{-\lambda}$. Since $\xi \in \mathfrak{k}, \theta\left(\xi_{\lambda}\right)=\xi_{-\lambda}$ and $z_{\lambda} \in \mathfrak{p}$. Moreover the vectors $z_{\lambda}$ are orthogonal to each other. Similarly $[\beta, \xi]=\sum_{\lambda \in \Delta_{+}} \lambda(\beta) z_{\lambda}$. So

$$
D^{2} \mu_{\mathfrak{p}}^{\beta}(x)(w, w)=-\sum_{\lambda(x)>0} \lambda(x) \lambda(\beta)\left|z_{\lambda}\right|^{2} .
$$

If there is $\lambda \in \Delta_{+}$such that $\lambda(x) \lambda(\beta)<0$, then $x$ is not a local maximum point. Otherwise the Hessian is negative semidefinite and $D^{2} \mu_{\mathfrak{p}}^{\beta}(x)(w, w)=0$ if and only if $z_{\lambda} \neq 0 \Rightarrow \lambda(\beta)=0$. This means that the kernel of $D^{2} \mu_{\mathfrak{p}}^{\beta}(x)$ is $\mathfrak{k}^{\beta} \cdot x=T_{x} \operatorname{Crit}\left(\mu_{\mathfrak{p}}^{\beta}\right)$. So the Hessian is degenerate only along the critical submanifold and is negative definite in the transverse direction. It follows that $x$ is a local maximum point. Summing up we have shown that $x$ is a local maximum point of $\mu_{\mathfrak{p}}^{\beta}$ if and only if $\lambda(x) \lambda(\beta) \geq 0$ for every $\lambda \in \Delta$. By Lemma 3.2 this is equivalent to the condition that $x$ and $\beta$ lie in the closure of some Weyl chamber. The result follows.

Proof of Proposition 3.1. We start assuming that $G$ is semisimple. Let $E$ be the set of all local maxima of $\mu_{\mathfrak{p}}^{\beta}$. Since the function $\mu_{\mathfrak{p}}^{\beta}$ is $K^{\beta}$-invariant, the sets $E$ and $\operatorname{Max}(\beta)$ are $K^{\beta}$-invariant. Since $\mathcal{O}$ is compact there is at least a point $x \in \operatorname{Max}(\beta)$. Let $\mathfrak{a} \subset \mathfrak{p}$ be a maximal subalgebra containing $x$ and $\beta$. If $y \in E$, then by Lemma 3.5 there are $a \in\left(K^{\beta}\right)^{0}$ and $\tilde{w} \in$ $W(\mathfrak{g}, \mathfrak{a})$, such that $y=a \cdot \tilde{w} \cdot x$. Since $y \in E$, also $\tilde{w} \cdot x \in E$. By Proposition 3.3 there are Weyl chambers $C, C^{\prime} \subset \mathfrak{a}$ such that $x, \beta \in \bar{C}$ and $w \cdot x, \beta \in$ $\overline{C^{\prime}}$. By Lemma 3.3 there is $w \in W$ such that $w \cdot x=\tilde{w} \cdot x$ and $w \cdot \beta=\beta$. By Proposition 3.2 there is $k \in\left(K^{\beta}\right)^{0}$ such that $w \cdot x=k \cdot x$. It follows that $y \in\left(K^{\beta}\right)^{0} \cdot x$. So $E \subset\left(K^{\beta}\right)^{0} \cdot x$. Since $\left(K^{\beta}\right)^{0} \cdot x \subset \operatorname{Max}(\beta) \subset E$ we conclude that $E=\operatorname{Max}(\beta)=\left(K^{\beta}\right)^{0} \cdot x$. In particular $\operatorname{Max}(\beta)$ is connected because it is an orbit of a connected group. Since $\operatorname{Max}(\beta)$ is $K^{\beta}$-stable we also have $\operatorname{Max}(\beta)=K^{\beta} \cdot x$. If $G$ is not semisimple, then split $\mathfrak{g}=\mathfrak{z} \oplus[\mathfrak{g}, \mathfrak{g}]$ with $\mathfrak{z}=\mathfrak{z}(\mathfrak{g})$. Accordingly $\mathfrak{p}=\mathfrak{z} \cap \mathfrak{p} \oplus \mathfrak{p}_{s s}, \mathfrak{k}=\mathfrak{k} \cap \mathfrak{z} \oplus \mathfrak{k}_{s s}$. Since $K$ is connected, $K=(Z(G) \cap K)^{0} \cdot K_{s s}$. If $\mathcal{O}=K \cdot x$ split $x=x_{0}+x_{1}$ with $x_{0} \in \mathfrak{z} \cap \mathfrak{p}$ and $x_{1} \in \mathfrak{p}_{s s}$. Then $\mathcal{O}=x_{0}+\mathcal{O}_{1}$ where $\mathcal{O}_{1}=K_{s s} \cdot x_{1}$. If $\beta \in \mathfrak{p}$, split $\beta=\beta_{0}+\beta_{1}$ with $\beta_{0} \in \mathfrak{p} \cap \mathfrak{z}$ and $\beta_{1} \in \mathfrak{p}_{s s}$. Then $\operatorname{Max}(\beta)=x_{0}+\operatorname{Max}\left(\beta_{1}\right)$. By Lemma 2.7 (b) $G_{s s}$ is a semisimple compatible subgroup of $U^{\mathbb{C}}$ and $\mathcal{O}_{1}$ is a $K_{s s}$-orbit in $\mathfrak{p}_{\text {ss }}$. Therefore we know that $\operatorname{Max}\left(\beta_{1}\right)$ is connected and that it is an orbit of both $\left(K_{s s}^{\beta_{1}}\right)^{0}$ and $K_{s s}^{\beta_{1}}$. Since $K^{\beta}=(Z(G) \cap K) \cdot K_{s s}^{\beta_{1}}$, we conclude that $\operatorname{Max}(\beta)$ is a connected orbit of $K^{\beta}$. Therefore it is also an orbit of $\left(K^{\beta}\right)^{0}$.

Corollary 3.1. Let $\beta$ be a non-zero vector in $\mathfrak{p}$ and let $F_{\beta}(\widehat{\mathcal{O}})$ be the exposed face of $\widehat{\mathcal{O}}$ defined by $\beta$, see (2.1). Then $\operatorname{ext} F_{\beta}(\widehat{\mathcal{O}})=\operatorname{Max}(\beta), F_{\beta}(\widehat{\mathcal{O}}) \subset \mathfrak{p}^{\beta}$ and $\operatorname{ext} F_{\beta}(\widehat{\mathcal{O}})$ is both a $K^{\beta}$ and a $\left(K^{\beta}\right)^{0}$-orbit. 
Proof. By Lemma $3.1 \operatorname{ext} F_{\beta}(\widehat{\mathcal{O}})=\mathcal{O} \cap F_{\beta}(\widehat{\mathcal{O}})=\operatorname{Max}(\beta)$. Since $\operatorname{Crit}\left(\mu_{\mathfrak{p}}^{\beta}\right)=$ $\mathcal{O} \cap \mathfrak{p}^{\beta}$, we see that $F_{\beta}(\widehat{\mathcal{O}}) \subset \mathfrak{p}^{\beta}$. By Proposition $3.1 \operatorname{ext} F_{\beta}(\widehat{\mathcal{O}})=\operatorname{Max}(\beta)$ is an orbit of $\left(K^{\beta}\right)^{0}$.

Proposition 3.4. Let $F$ be a non-empty face of $\widehat{\mathcal{O}}$. Then there is an abelian subalgebra $\mathfrak{s} \subset \mathfrak{p}$ such that $F$ is an orbitope of $\left(G^{\mathfrak{s}}\right)^{0}$, i.e., $F \subset \mathfrak{z}_{\mathfrak{p}}(\mathfrak{s})$ and $\operatorname{ext} F$ is an orbit of $\left(K^{\mathfrak{s}}\right)^{0}$. If $F$ is proper, then $\mathfrak{s} \neq\{0\}$.

Proof. Fix a chain of faces $F=F_{0} \subsetneq F_{1} \subsetneq \cdots \subsetneq F_{k}=\widehat{\mathcal{O}}$, such that for any $i$ there is no face strictly contained between $F_{i-1}$ and $F_{i}$. This is possible by Lemma 2.5. We will prove the result by induction on $k$. If $k=0$, then $F=\widehat{\mathcal{O}}$, so it is enough to set $\mathfrak{s}=\{0\}$. Let $k>1$ and assume that the theorem is proved for faces contained in a maximal chain of length $k-1$. Fix $F$ with a maximal chain as above of length $k$. By the inductive hypothesis the theorem holds for $F_{1}$, so there is a non-trivial abelian subalgebra $\mathfrak{s}_{1} \subset \mathfrak{p}$ such that $F_{1} \subset \mathfrak{p}^{\mathfrak{s}_{1}}$ and ext $F_{1}$ is an orbit of $\left(K^{\mathfrak{s}_{1}}\right)^{0}$. In other words $F_{1}$ is an orbitope of $\left(G^{\mathfrak{s}_{1}}\right)^{0}$, which is a compatible subgroup by Lemma 2.7 (c). Since $F$ is a maximal face of $F_{1}$, it is exposed. There is $\beta \in \mathfrak{p}^{\mathfrak{s}_{1}}$ such that $F=F_{\beta}\left(F_{1}\right)$. Set $\mathfrak{s}=\mathfrak{s}_{1} \oplus \mathbb{R} \beta$. By Corollary $3.1 F \subset\left(\mathfrak{p}^{\mathfrak{s}}\right)^{\beta}=\mathfrak{p}^{\mathfrak{s}}$ and $\operatorname{ext} F$ is an orbit of $\left(\left(K^{\mathfrak{s}_{1}}\right)^{\beta}\right)^{0}=\left(K^{\mathfrak{s}}\right)^{0}$. Thus, the inductive step is completed. If $\mathfrak{s}=\{0\}$, then $\left(K^{\mathfrak{s}}\right)^{0}=K$, ext $F=\mathcal{O}$ and $F=\widehat{\mathcal{O}}$. So for proper faces $\mathfrak{s} \neq\{0\}$.

\subsection{All faces are exposed}

Let $G \subset U^{\mathbb{C}}$ be a compatible subgroup and let $\mathcal{O}$ be a $K$-orbit in $\mathfrak{p}$. In general $\operatorname{dim} \widehat{\mathcal{O}}$ might be less than $\operatorname{dim} \mathfrak{p}$ and there might be some normal subgroup of $K$ that acts trivially on $\mathcal{O}$. We wish to describe a decomposition of $G$ that is useful in dealing with this degeneracy. Let $A$ be the affine hull of $\mathcal{O}$. This is an affine subspace of $\mathfrak{p}$ and we can write $A=x_{0}+\mathfrak{p}_{1}$, where $\mathfrak{p}_{1} \subset \mathfrak{p}$ is a linear subspace and $x_{0} \in \mathfrak{p}$. If we impose that $x_{0} \perp \mathfrak{p}_{1}$, then $x_{0}$ is uniquely determined. It follows that $x_{0}$ is fixed by $K$. Hence by Lemma $2.2 x_{0} \in \operatorname{relint} \widehat{\mathcal{O}}$. Set also

$$
\mathfrak{k}_{1}:=\left[\mathfrak{p}_{1}, \mathfrak{p}_{1}\right] \quad \mathfrak{p}_{0}=\mathfrak{p}_{1}^{\perp} \quad \mathfrak{k}_{0}=\mathfrak{k}_{1}^{\perp} \quad \mathfrak{g}_{1}:=\mathfrak{k}_{1} \oplus \mathfrak{p}_{1} \quad \mathfrak{g}_{0}:=\mathfrak{k}_{0} \oplus \mathfrak{p}_{0} .
$$

Thus $\mathfrak{k}=\mathfrak{k}_{0} \oplus \mathfrak{k}_{1}$ and $\mathfrak{p}=\mathfrak{p}_{0} \oplus \mathfrak{p}_{1}$ and $\mathfrak{g}=\mathfrak{g}_{0} \oplus \mathfrak{g}_{1}$.

Proposition 3.5. $\mathfrak{g}_{1}$ is a semisimple ideal of $\mathfrak{g}$ and $\mathfrak{g}_{0}$ is a reductive ideal. If $G_{1}, K_{0}, K_{1}$ are the corresponding analytic (connected) subgroups, then $G_{1}$ is compatible with $U^{\mathbb{C}}$ and $K^{0}=K_{0} \cdot K_{1}$. If $x \in \mathcal{O}$, then $x=x_{0}+x_{1}$ for some $x_{1} \in \mathfrak{p}_{1}$ and $\mathcal{O}=x_{0}+K_{1} \cdot x_{1}$. 
Proof. Since $\mathcal{O}$ is a $K$-orbit, its affine hull is $K$-invariant. Therefore $x_{0}$ is fixed by $K$ and $\left[\mathfrak{k}, \mathfrak{p}_{1}\right] \subset \mathfrak{p}_{1}$. It follows that $\left[\mathfrak{k}, \mathfrak{k}_{1}\right]=\left[\mathfrak{k},\left[\mathfrak{p}_{1}, \mathfrak{p}_{1}\right]\right]=\left[\mathfrak{p}_{1},\left[\mathfrak{p}_{1}, \mathfrak{k}\right]\right] \subset$ $\left[\mathfrak{p}_{1}, \mathfrak{p}_{1}\right]=\mathfrak{k}_{1}$. Since $\left[\mathfrak{k}, \mathfrak{p}_{1}\right] \subset \mathfrak{p}_{1}$ and $\left[\mathfrak{k}, \mathfrak{k}_{1}\right] \subset \mathfrak{k}_{1}$ also $\left[\mathfrak{k}, \mathfrak{p}_{0}\right] \subset \mathfrak{p}_{0}$ and $\left[\mathfrak{k}, \mathfrak{k}_{0}\right] \subset \mathfrak{k}_{0}$. Moreover $\left\langle\left[\mathfrak{p}_{1}, \mathfrak{p}_{0}\right], \mathfrak{k}\right\rangle=B\left(\left[\mathfrak{p}_{1}, \mathfrak{p}_{0}\right], \mathfrak{k}\right)=B\left(\mathfrak{p}_{0},\left[\mathfrak{k}, \mathfrak{p}_{1}\right]\right) \subset B\left(\mathfrak{p}_{0}, \mathfrak{p}_{1}\right)=\left\langle\mathfrak{p}_{0}, \mathfrak{p}_{1}\right\rangle=$ 0. ( $B$ is the bilinear form defined at the end of Section 2.2.) Since $\left[\mathfrak{p}_{1}, \mathfrak{p}_{0}\right] \subset \mathfrak{k}$ this means that $\left[\mathfrak{p}_{1}, \mathfrak{p}_{0}\right]=0$. Using the Jacobi identity we get also $\left[\mathfrak{p}_{0}, \mathfrak{k}_{1}\right]=$ $\left[\mathfrak{p}_{0},\left[\mathfrak{p}_{1}, \mathfrak{p}_{1}\right]\right]=\left[\mathfrak{p}_{1},\left[\mathfrak{p}_{1}, \mathfrak{p}_{0}\right]\right]=0$. Set $\mathfrak{g}_{1}:=\mathfrak{k}_{1} \oplus \mathfrak{p}_{1}$. We have just showed that $\mathfrak{g}_{1}$ is an ideal of $\mathfrak{g}$. Since it is $\theta$-invariant, $\mathfrak{g}_{1}$ is a reductive subalgebra. We claim that it is semisimple. $\mathfrak{k}_{1} \subset\left[\mathfrak{g}_{1}, \mathfrak{g}_{1}\right]$, so $\mathfrak{z}\left(\mathfrak{g}_{1}\right) \subset \mathfrak{p}_{1}$. Pick $x \in \mathcal{O}$. We can split $x=x_{0}+x_{1}+x_{2}$ where $x_{0}$ is as above, $x_{2} \in \mathfrak{z}\left(\mathfrak{g}_{1}\right) \cap \mathfrak{p}_{1}, x_{1} \in \mathfrak{p}_{1}$ and $x_{1} \perp \mathfrak{z}\left(\mathfrak{g}_{1}\right)$. It follows that $\mathcal{O}=x_{0}+x_{2}+K \cdot x_{1}$, so the affine hull of $\mathcal{O}$ is $x_{0}+x_{2}+\mathfrak{p}_{1} \cap \mathfrak{z}\left(\mathfrak{g}_{1}\right)^{\perp}$. Therefore $x_{2}=0$ and $\mathfrak{p}_{1} \cap \mathfrak{z}\left(\mathfrak{g}_{1}\right)^{\perp}=\mathfrak{p}_{1}$, i.e., $\mathfrak{z}\left(\mathfrak{g}_{1}\right)=$ $\{0\}$. This proves that $\mathfrak{g}_{1}$ is semisimple. Let $G_{1} \subset G$ the (connected) analytic subgroup tangent to $\mathfrak{g}_{1}$. It is normal, closed [16, p. 440] and compatible by Lemma 2.7 (c). The $B$-orthogonal complement of $\mathfrak{g}_{1}$ is $\mathfrak{k}_{0} \oplus \mathfrak{p}_{0}$, which is also an ideal. So $K=K_{0} \cdot K_{1}$ where $K_{1}=G_{1} \cap U$ and $K_{0}$ is the analytic subgroup of $K$ tangent to $\mathfrak{k}_{0}$. Since $K_{0}$ and $K_{1}$ are normal commuting subgroups $K_{0}$ acts trivially on $\mathfrak{p}_{1}$. Hence, $\mathcal{O}=x_{0}+K_{1} \cdot x_{1}$.

This decomposition can be further refined by setting $\mathfrak{g}_{2}:=\left[\mathfrak{g}_{0}, \mathfrak{g}_{0}\right]$ and $\mathfrak{g}_{3}:=\mathfrak{z}(\mathfrak{g})=\mathfrak{z}\left(\mathfrak{g}_{0}\right)$. They are both $\theta$-invariant ideals of $\mathfrak{g}, \mathfrak{g}_{2}$ is semisimple and

$$
\mathfrak{g}=\mathfrak{g}_{1} \stackrel{\perp}{\oplus} \mathfrak{g}_{2} \stackrel{\perp}{\oplus} \mathfrak{g}_{3}
$$

Set $\mathfrak{p}_{i}:=\mathfrak{g}_{i} \cap \mathfrak{p}$ and $\mathfrak{k}_{i}:=\mathfrak{g}_{i} \cap \mathfrak{k}$. At the group level $K^{0}=K_{1} \cdot K_{2} \cdot K_{3}$, where $K_{i}$ are the corresponding analytic (connected) subgroups. Since $K \cdot x_{0}=x_{0}$, $x_{0} \in \mathfrak{g}_{3}$.

Let $\mathfrak{a} \subset \mathfrak{p}$ be a maximal subalgebra. Let $\pi: \mathfrak{p} \rightarrow \mathfrak{a}$ denote the orthogonal projection. Set

$$
P:=\pi(\mathcal{O}) .
$$

The following convexity theorem of Kostant [18] is the basic ingredient in the whole theory.

Theorem 3.1 (Kostant). Let $x \in \mathfrak{a} \cap \mathcal{O}$. Then $P=\operatorname{conv}(W \cdot x)$. In particular, $P$ is a convex polytope, ext $P=\mathcal{O} \cap \mathfrak{a}$ and $\operatorname{ext} P$ is a $W$-orbit.

The original proof of Kostant assumes that $G$ is semisimple. One easily reduces to that case using Proposition 3.5. The theorem can be proved 
within the framework of the gradient momentum map [9, Rmk. 5.4]. Another approach is by observing that the orbits of polar representations are isoparametric submanifolds. Terng [25] has proved a convexity theorem for isoparametric submanifolds, which in the case of polar orbits gives the original statement by Kostant. See also [21]. The following lemma is a consequence of Kostant convexity theorem. See [7, Lemma 7] for a proof.

Lemma 3.6. (i) If $E \subset \mathfrak{p}$ is a $K$-invariant convex subset, then $E \cap \mathfrak{a}=$ $\pi(E)$. (ii) If $A \subset \mathfrak{a}$ is a $W$-invariant convex subset, then $K \cdot A$ is convex and $\pi(K \cdot A)=A$.

Proposition 3.6. Let $F$ be a face of $\widehat{\mathcal{O}}$. Choose a subalgebra $\mathfrak{s} \subset \mathfrak{p}$ such that $F$ be an orbitope of $\left(G^{\mathfrak{s}}\right)^{0}$. Let $\mathfrak{a}$ be a maximal subalgebra of $\mathfrak{p}$ containing s. Set $\sigma:=\pi(\operatorname{ext} F)$. Then $\sigma=\pi(F)=F \cap \mathfrak{a}$ and $\sigma$ is a non-empty face of the polytope $P$. If $F$ is proper, then $\sigma$ is proper. $F$ is an orbitope of $\left(G^{\sigma^{\perp}}\right)^{0}$, where $\sigma^{\perp} \subset \mathfrak{a}$ denotes the orthogonal to the tangent space of $\sigma$. Moreover ext $F$ is an orbit of $K^{\sigma^{\perp}}$ and $F=K^{\sigma^{\perp}} \cdot \sigma$.

Proof. The set ext $F$ is an orbit of $\left(K^{\mathfrak{s}}\right)^{0}$ and $\mathfrak{a} \subset \mathfrak{g}^{\mathfrak{s}}$. By Kostant theo$\operatorname{rem} \pi(\operatorname{ext} F)=\operatorname{conv}(\operatorname{ext} F \cap \mathfrak{a})$ and $\operatorname{ext} F \cap \mathfrak{a}$ is an orbit of the Weyl group $W=W\left(\mathfrak{g}^{\mathfrak{s}}, \mathfrak{a}\right)$. So $\sigma$ is convex. Fix $x \in \operatorname{ext} F \cap \mathfrak{a}$. Since $\pi$ is linear, $\pi(F) \subset$ $\operatorname{conv}(\pi(\operatorname{ext} F))=\sigma$. On the other hand $\operatorname{ext} \sigma \subset W \cdot x=(\operatorname{ext} F) \cap \mathfrak{a}$. Hence $\sigma \subset F \cap \mathfrak{a}$. And obviously $F \cap \mathfrak{a} \subset \pi(F)$. Summing up $\pi(F) \subset \sigma \subset F \cap \mathfrak{a} \subset$ $\pi(F)$. The first assertion is proved. That $\sigma$ is a face of $P$ follows directly from Lemma 2.6, while $\sigma=\pi(F) \neq \emptyset$ since $F \neq \emptyset$. To check the other assertions observe that ext $F$ is an orbit of $\left(K^{\mathfrak{s}}\right)^{0}$, so that we can apply Proposition 3.5 to this orbit. We get a semisimple normal subgroup $G_{1}$ of $\left(G^{\mathfrak{s}}\right)^{0}$, a decomposition $\mathfrak{g}^{\mathfrak{s}}=\mathfrak{g}_{1} \oplus \mathfrak{g}_{2} \oplus \mathfrak{g}_{3}$ like (3.1) and compact subgroups $K_{1}$, $K_{2}, K_{3}=Z\left(K^{\mathfrak{s}}\right)^{0}$ such that $\left(K^{\mathfrak{s}}\right)^{0}=K_{1} \cdot K_{2} \cdot K_{3}$. It follows that $\mathfrak{a}=\mathfrak{a}_{1} \oplus$ $\mathfrak{a}_{2} \oplus \mathfrak{p}_{3}$, where $\mathfrak{a}_{i}:=\mathfrak{a} \cap \mathfrak{g}_{i}$ is a maximal subalgebra of $\mathfrak{p}_{i}$ for $i=1,2$. Moreover ext $F=x_{0}+K_{1} \cdot x_{1}$, the affine hull of $F$ is $x_{0}+\mathfrak{p}_{1}$ and $x_{0} \in \operatorname{relint} F$. The restriction of $\pi$ to $\mathfrak{p}_{1}$ is the orthogonal projection $\mathfrak{p}_{1} \rightarrow \mathfrak{a}_{1}$ and the affine hull of $\sigma$ is $x_{0}+\mathfrak{a}_{1}$. Hence $\sigma^{\perp}=\mathfrak{a}_{2} \oplus \mathfrak{p}_{3} \cdot \mathfrak{g}_{1}$ is semisimple and centralizes. Thus $\mathfrak{s} \subset \sigma^{\perp}, K^{\sigma^{\perp}} \subset K^{\mathfrak{s}}$ and $\left(K^{\sigma^{\perp}}\right)^{0}=K_{1} \cdot K_{3}$. So $K_{1} \subset K^{\sigma^{\perp}} \subset K^{\mathfrak{s}}$ and $K_{1} \cdot x \subset K^{\sigma^{\perp}} \cdot x \subset K^{\mathfrak{s}} \cdot x$. Since $K_{1} \cdot x=K^{\mathfrak{s}} \cdot x=\operatorname{ext} F$ we get that ext $F$ is an orbit of $K^{\sigma^{\perp}}$. But ext $F$ is connected, so it is also an orbit of $\left(K^{\sigma^{\perp}}\right)^{0}$. Since $\sigma^{\perp}=\mathfrak{a}_{2} \oplus \mathfrak{p}_{3}, x_{0}+\mathfrak{p}_{1} \subset \mathfrak{p}_{3} \oplus \mathfrak{p}_{1}=\mathfrak{p}^{\sigma^{\perp}}$. This shows that $F$ is an orbitope of $\left(G^{\sigma^{\perp}}\right)^{0}$. We have to prove that $F=K^{\sigma^{\perp}} \cdot \sigma$. Since $K_{2}$ acts trivially on $x_{0}+\mathfrak{p}_{1}, K^{\sigma^{\perp}} \cdot \sigma=K^{\mathfrak{s}} \cdot \sigma$. Since $F$ is $K^{\mathfrak{s}}$-invariant, we get 
$K^{\sigma^{\perp}} \cdot \sigma \subset F$. On the other hand, ext $F \subset K^{\mathfrak{s}} \cdot \sigma$. Since $\sigma$ is $W$-invariant we can apply Lemma 3.6 (with $K=K^{\mathfrak{s}}$ and $\mathfrak{p}=\mathfrak{p}^{\mathfrak{s}}$ ) to get that $K^{\mathfrak{s}} \cdot \sigma$ is convex. Therefore we get $F=K^{\mathfrak{s}} \cdot \sigma=K^{\sigma^{\perp}} \cdot \sigma$. It remains to prove that $\sigma$ is proper, when $F$ is proper. Assume first that the affine hull $\widehat{\mathcal{O}}$ is $\mathfrak{p}$. Then the affine hull of $P$ is $\mathfrak{a}$. If $F$ is proper, then $\mathfrak{s} \neq\{0\}$, so $\mathfrak{a}_{1} \subsetneq \mathfrak{a}$ and $\sigma \subsetneq P$. In the general case, we have to apply Proposition 3.5 this time to $\mathcal{O}$ rather than ext $F$. $\widehat{\mathcal{O}}$ turns out to be a translate of an orbitope of a semisimple subgroup of $G$ by an element of the center of $\mathfrak{g}$. $\mathfrak{a}$ splits into the center of $\mathfrak{g}$ and a maximal subalgebra of the semisimple subgroup. With this we easily reduce to the case we have just considered.

Corollary 3.2. Let $F_{1}, F_{2}$ be proper faces of $\widehat{\mathcal{O}}$, and let $\mathfrak{s}_{1}, \mathfrak{s}_{2} \subset \mathfrak{p}$ be subalgebras such that $F_{i}$ is a $\left(G^{\mathfrak{s}_{i}}\right)^{0}$-orbitope. Assume that $\mathfrak{a} \subset \mathfrak{p}$ is a maximal subalgebra containing both $\mathfrak{s}_{1}$ and $\mathfrak{s}_{2}$. If $F_{1} \cap \mathfrak{a}=F_{2} \cap \mathfrak{a}$, then $F_{1}=F_{2}$.

Proof. If $\sigma:=F_{i} \cap \mathfrak{a}$, then $F_{1}=K^{\sigma^{\perp}} \cdot \sigma=F_{2}$.

Theorem 3.2. All proper faces of $\widehat{\mathcal{O}}$ are exposed.

Proof. Given a proper face $F \subset \widehat{\mathcal{O}}$ choose a subalgebra $\mathfrak{s} \subset \mathfrak{p}$ such that $F$ be a $\left(G^{\mathfrak{s}}\right)^{0}$-orbitope and choose a maximal subalgebra $\mathfrak{a} \subset \mathfrak{p}$ containing $\mathfrak{s}$. By Proposition $3.6 \sigma:=F \cap \mathfrak{a}$ is a proper face of $P$. Since all faces of a polytope are exposed $\left[24\right.$, p. 95], there is a vector $\beta \in \mathfrak{a}$ such that $\sigma=F_{\beta}(P)$. Since $\beta \in \mathfrak{a}$ and $P=\pi(\mathcal{O}), h_{P}(\beta)=\max _{x \in \mathcal{O}}\langle\beta, x\rangle=h_{\widehat{\mathcal{O}}}(\beta)$. Set $F^{\prime}:=F_{\beta}(\widehat{\mathcal{O}})$. We wish to show that $F=F^{\prime}$. The inclusion $F \subset F^{\prime}$ is immediate: if $x \in F$, then $\pi(x) \in \sigma$, so $\langle x, \beta\rangle=h_{P}(\beta)=h_{\widehat{\mathcal{O}}}(\beta)$. It is also immediate that $F^{\prime} \cap \mathfrak{a}=\sigma$. So we have two faces $F$ and $F^{\prime}$ with $F \cap \mathfrak{a}=F^{\prime} \cap \mathfrak{a}=\sigma$. Set $\mathfrak{s}^{\prime}:=\mathbb{R} \beta \subset \mathfrak{a}$. By Corollary $3.1 F^{\prime}$ is an orbitope of $\left(G^{\mathfrak{s}^{\prime}}\right)^{0}$. Applying Corollary 3.2 we get $F=F^{\prime}=F_{\beta}(\widehat{\mathcal{O}})$.

Corollary 3.3. If $\mathcal{O}^{\prime} \subset \mathcal{O}$ is a smooth submanifold, then $\operatorname{conv}\left(\mathcal{O}^{\prime}\right)$ is a face of $\widehat{\mathcal{O}}$ if and only if there is a vector $\beta$ such that $\mathcal{O}^{\prime}=\operatorname{Max}(\beta)$.

Proof. Set $F=\operatorname{conv}\left(\mathcal{O}^{\prime}\right)$. From the fact that $\mathcal{O}$ is contained in a sphere, it follows as in Lemma 3.1 that ext $F=\mathcal{O}^{\prime}$. Therefore the statement follows immediately from the fact that every face of $\widehat{\mathcal{O}}$ is exposed and from Lemma 3.1. 


\subsection{Faces and parabolic subgroups}

In this section, we prove Theorem 1.2, which follows from Propositions 3.8 and 3.9 below. Given a face $F \subset \widehat{\mathcal{O}}$ set

$$
\begin{aligned}
& H_{F}:=\{g \in K: g F=F\}=\{g \in K: g \cdot \operatorname{ext} F=\operatorname{ext} F\} \\
& Q_{F}:=\{g \in G: g \cdot \operatorname{ext} F=\operatorname{ext} F\} \\
& C_{F}:=\left\{\beta \in \mathfrak{p}: F=F_{\beta}(\widehat{\mathcal{O}})\right\} .
\end{aligned}
$$

Denote by $C_{F}^{H_{F}}$ the vectors of $C_{F}$ that are fixed by $H_{F}$.

Proposition 3.7. For any face $F$ the set ext $F$ is an orbit of $H_{F}$. If $F$ is proper, then $C_{F}^{H_{F}} \neq \emptyset$. For any $\beta \in C_{F}^{H_{F}}, H_{F}=K^{\beta}$ and $F \subset \mathfrak{p}^{\beta}$.

Proof. The group $H_{F}$ is compact. By Proposition 3.4 ext $F$ is an orbit of some subgroup $K^{\prime} \subset K$. Hence $K^{\prime} \subset H_{F}$ and ext $F$ is an orbit also of $H_{F}$. It follows that $H_{F}$ preserves both $\widehat{\mathcal{O}}$ and $F$, so by Lemma 2.3 there is a vector $\beta \in C_{F}$ that is fixed by $H_{F}$. This proves that $C_{F}^{H_{F}} \neq \emptyset$. On the other hand given any $\beta \in C_{F}^{H_{F}}$, we have $H_{F} \subset K^{\beta}$ and $F=F_{\beta}(\widehat{\mathcal{O}})$. By Lemma 3.1, $F \subset \mathfrak{p}^{\beta}$ and ext $F=K^{\beta} \cdot x$. It follows that $K^{\beta} \subset H_{F}$, hence $H_{F}=K^{\beta}$.

Lemma 3.7. Let $\mathfrak{q}_{1}, \mathfrak{q}_{2}$ be subalgebras of $\mathfrak{g}$. Assume that $\mathfrak{q}_{1}$ is parabolic, that $\mathfrak{q}_{1} \subset \mathfrak{q}_{2}$ and that $\mathfrak{q}_{1} \cap \mathfrak{k}=\mathfrak{q}_{2} \cap \mathfrak{k}$. Then $\mathfrak{q}_{1}=\mathfrak{q}_{2}$.

Proof. Assume that $\mathfrak{q}_{1}=\mathfrak{g}^{\beta+}$ for some $\beta \in \mathfrak{p}$. Then $\mathfrak{q}_{1} \cap \mathfrak{k}=\mathfrak{k}^{\beta}$. Denote by $V_{\lambda}$ the eigenspace of $\operatorname{ad} \beta$ with eigenvalue $\lambda$. Then $\mathfrak{q}_{1}=\bigoplus_{\lambda \in J} V_{\lambda}$ where $J$ is the set of non-negative eigenvalues of $\operatorname{ad} \beta$. Since $\beta \in \mathfrak{q}_{1} \subset \mathfrak{q}_{2}, \mathfrak{q}_{2}$ is $\operatorname{ad} \beta$ stable. We have

$$
\mathfrak{q}_{2}=\bigoplus_{\lambda \in I}\left(V_{\lambda} \cap \mathfrak{q}_{2}\right)
$$

for some set of eigenvalues $I$ and we can assume that $V_{\lambda} \cap \mathfrak{q}_{2} \neq\{0\}$ for every $\lambda \in I$. We wish to prove that $I \subset[0, \infty)$. If not, there would be some negative $\lambda \in I$. Pick a non-zero $\xi \in V_{\lambda} \cap \mathfrak{q}_{2}$. Then $\theta(\xi) \in V_{-\lambda} \subset \mathfrak{q}_{1} \subset \mathfrak{q}_{2}$. So $\xi+$ $\theta(\xi) \in \mathfrak{q}_{2} \cap \mathfrak{k}$. By assumption $\mathfrak{q}_{2} \cap \mathfrak{k}=\mathfrak{q}_{1} \cap \mathfrak{k}=\mathfrak{g}^{\beta+} \cap \mathfrak{k}=\mathfrak{k}^{\beta}$. So we should have $[\beta, \xi+\theta(\xi)]=0$, while $\quad[\beta, \xi+\theta(\xi)]=\lambda(\xi-\theta(\xi)) \neq 0 . \quad$ The contradiction shows that $I \subset[0, \infty)$. So $I \subset J$ and $\mathfrak{q}_{2} \subset \mathfrak{q}_{1}$.

Proposition 3.8. If $F \subset \widehat{\mathcal{O}}$ is a proper face, and $\beta \in C_{F}^{H_{F}}$, then $Q_{F}=$ $G^{\beta+}$. 
Proof. We prove first that $G^{\beta+} \subset Q_{F}$, i.e., that $G^{\beta+}$ preserves ext $F$. Since $\beta \in C_{F}^{H_{F}}, H_{F}=K^{\beta}$. In general $G^{\beta+}$ will not be connected. Nevertheless $K \cap$ $G^{\beta+}=K^{\beta}$ meets all components of $G^{\beta+}$. By Proposition $3.7 K^{\beta}=H_{F} \subset$ $Q_{F}$. So it is enough to prove that $\left(G^{\beta+}\right)^{0} \subset Q_{F}$. This amounts to showing that for any $\xi \in \mathfrak{g}^{\beta+}$ the vector field $\xi_{\mathcal{O}}$ is tangent to ext $F$. Fix an arbi$\operatorname{trary} x \in \operatorname{ext} F$. Since $F=F_{\beta}(\widehat{\mathcal{O}})$, ext $F=\operatorname{Max}(\beta)$, so $x$ is a maximum point of $\mu_{\mathfrak{p}}^{\beta}$. Hence $V_{+}=\{0\}$ in (2.6). By Proposition $2.1 d \alpha_{e}\left(\mathfrak{g}^{\beta+}\right)=d \alpha_{e}\left(\mathfrak{g}^{\beta}\right)+$ $d \alpha_{e}\left(\mathfrak{r}_{+}^{\beta}\right) \subset V_{0}+V_{+}=V_{0}$. Hence for any $\xi \in \mathfrak{g}^{\beta+}, \xi_{\mathcal{O}}(x)=d \alpha_{e}(\xi) \in V_{0}=$ $T_{x}$ ext $F$. Thus we proved that $G^{\beta+} \subset Q_{F}$. We also know that $G^{\beta+} \cap K=$ $K^{\beta}=H_{F}=Q_{F} \cap K$. Also, $Q_{F} \subset G$ is a closed subgroup, hence a Lie subgroup. Thus we can apply Lemma 3.7 to the Lie algebras of $G^{\beta+}$ and $Q_{F}$, respectively, and we obtain $\mathfrak{g}^{\beta+}=\mathfrak{q}_{F}$. Therefore $Q_{F} \subset N_{G}\left(\mathfrak{q}_{F}\right)=G^{\beta+}$. And thus the theorem is proved.

Proposition 3.9. The set $\{\operatorname{ext} F: F$ a non-empty face of $\widehat{\mathcal{O}}\}$ coincides with the set of all closed orbits of parabolic subgroups of $G$. Any parabolic subgroup $Q \subset G$ has a unique closed orbit, which equals the set of extreme points of a unique face of $F \subset \widehat{\mathcal{O}}$. If $Q=G^{\beta+}$, then $F=F_{\beta}(\widehat{\mathcal{O}})$.

Proof. Let $Q \subset G$ be parabolic. There is at least one closed orbit since the action is algebraic. Choose $\beta \in \mathfrak{p}$ such that $Q=G^{\beta+}$. Then $K^{\beta}=Q \cap K$. Let $\mathcal{O}^{\prime}$ be any closed orbit of $Q$ and let $x \in \mathcal{O}^{\prime}$ be a maximum point of $\mu_{\mathfrak{p}}^{\beta}$ over $\mathcal{O}^{\prime}$. Since the gradient of $\mu_{\mathfrak{p}}^{\beta}$ at $x$ is $\beta_{\mathcal{O}}(x)$ and $\beta \in \mathfrak{g}^{\beta+}$, we get $\beta_{\mathcal{O}}(x)=0$. By Proposition $2.1 d \alpha_{e}\left(\mathfrak{g}^{\beta+}\right)=V_{0} \oplus V_{+}$, so $V_{+} \subset T_{x}\left(G^{\beta+} \cdot x\right)=$ $T_{x} \mathcal{O}^{\prime}$. Since $x$ is a maximum point of $\mu_{\mathfrak{p}}^{\beta}$ over $\mathcal{O}^{\prime}$, we conclude that $V_{+}=\{0\}$. Thus, $x$ is a local maximum point of $\mu_{\mathfrak{p}}^{\beta}$ and $R^{\beta+}$ acts trivially on $\mathcal{O}^{\prime}$. But $\mu_{\mathfrak{p}}^{\beta}$ has only global maxima; hence $x \in \operatorname{Max}(\beta)$ and $\mathcal{O}^{\prime}=G^{\beta} \cdot x=K^{\beta} \cdot x=$ $\operatorname{Max}(\beta)$. Set $F=F_{\beta}(\widehat{\mathcal{O}})$. Then $\mathcal{O}^{\prime}=\operatorname{ext} F$. This proves that the closed orbit is unique.

Corollary 3.4. For any face $F$ we have $C_{F}^{H_{F}}=\left\{\beta \in \mathfrak{p}: G^{\beta+}=Q_{F}\right\}$.

Proof. By Proposition 3.8 the set on the left is included in the set on the right. Conversely, if $\beta$ is in the set on the right, then $\beta \in C_{F}$ with $F=F_{\beta}(\widehat{\mathcal{O}})$, by the previous Theorem. Since $H_{F}=Q_{F} \cap K=G^{\beta+} \cap K=K^{\beta}, \beta$ is also fixed by $H_{F}$.

If $F$ is a proper face set

$$
\mathfrak{s}_{F}:=\operatorname{span}\left(C_{F}^{H_{F}}\right) \quad G_{F}:=Q_{F} \cap \theta\left(Q_{F}\right) .
$$


If $\beta \in C_{F}^{H_{F}}$, then $G_{F}:=G^{\beta}$.

Corollary 3.5. $\mathfrak{s}_{F}$ is an abelian subalgebra of $\mathfrak{p}$ and $\mathfrak{s}_{F}=\mathfrak{z}\left(\mathfrak{g}_{F}\right) \cap \mathfrak{p}$.

Proof. $\mathfrak{s}_{F}$ is the span of $C_{F}^{H_{F}}$ and $\mathfrak{g}_{F}=\mathfrak{q}_{F} \cap \theta \mathfrak{q}_{F}$. Thus, the result follows from Corollary 3.4 and Lemma 2.8.

Corollary 3.6. $H_{F}=K^{\mathfrak{s}_{F}}$ and $G_{F}=G^{\mathfrak{s}_{F}}$.

Proof. It follows from the discussion in the proof of Lemma 2.8, that the vectors of $C_{F}^{H_{F}}$ are regular in $\mathfrak{s}_{F}=\mathfrak{a}_{I}$, i.e., if a root vanishes on $\beta \in C_{F}^{H_{F}}$, then it vanishes on the whole of $\mathfrak{s}_{F}$. Thus, $K^{\mathfrak{s}_{F}}=K^{\beta}$ and $G^{\mathfrak{s}_{F}}=G^{\beta}$.

Corollary 3.7. The face $F$ is an orbitope of $G_{F}^{0}$.

Proof. If $\beta \in C_{F}^{H_{F}}$, then $F$ is a $\left(G^{\beta}\right)^{0}$-orbitope by Corollary 3.1.

Corollary 3.8. Let $F$ be a face and let $\mathfrak{a} \subset \mathfrak{p}$ be a maximal subalgebra. Then $C_{F}^{H_{F}} \cap \mathfrak{a} \neq \emptyset$ if and only if $C_{F}^{H_{F}} \subset \mathfrak{a}$ if and only if $\mathfrak{a} \subset \mathfrak{g}_{F}$.

Proof. If $\beta \in C_{F}^{H_{F}} \cap \mathfrak{a}$, then $[\beta, \mathfrak{a}]=0$. Since $\beta$ is regular in $\mathfrak{s}_{F}$, we get $\mathfrak{s}_{F} \subset \mathfrak{a}$. Conversely, if $\mathfrak{s}_{F} \subset \mathfrak{a}$, then $C_{F}^{H_{F}} \subset \mathfrak{a}$. Since $\mathfrak{g}_{F}=\mathfrak{g}^{\mathfrak{s}_{F}}$ the condition $\mathfrak{s}_{F} \subset \mathfrak{a}$ is equivalent to $\mathfrak{a} \subset \mathfrak{g}_{F}$.

\subsection{Proof of Theorem 1.1}

Fix a maximal subalgebra $\mathfrak{a} \subset \mathfrak{p}$. Denote by $\mathscr{F}(\widehat{\mathcal{O}})$ the set of proper faces of $\mathcal{O}$ and by $\mathscr{F}(P)$ the set of proper faces of the polytope $P$. If $F$ is a face of $\mathcal{O}$ and $a \in K$, then $a \cdot F$ is still a face, so $K$ acts on $\mathscr{F}(\widehat{\mathcal{O}})$. Similarly $W=W(\mathfrak{g}, \mathfrak{a})$ acts on $\mathscr{F}(P)$. We wish to show that $\mathscr{F}(\widehat{\mathcal{O}}) / K \cong \mathscr{F}(P) / W$.

Lemma 3.8. For every face of $\widehat{\mathcal{O}}$ there is $a \in K$ such that $\mathfrak{s}_{a \cdot F} \subset \mathfrak{a}$. The face $a \cdot F$ is unique up to $N_{K}(\mathfrak{a})$.

Proof. By Theorem $3.2 F=F_{\gamma}(\widehat{\mathcal{O}})$ and $H_{F}=K^{\gamma}$ for some $\gamma \in \mathfrak{p}$. Choose $a \in K$ such that $\operatorname{Ad}(a) \gamma \in \mathfrak{a}$. Then $a \cdot F=F_{\operatorname{Ad}(a) \gamma}(\widehat{\mathcal{O}})$. Therefore $\operatorname{Ad}(a) \gamma$ belongs to $C_{a \cdot F}^{H_{a \cdot F}}$ and also to $\mathfrak{a}$. By Corollary $3.6 \mathfrak{s}_{a \cdot F} \subset \mathfrak{a}$. To prove the second statement it is enough to show that if $F=F_{\gamma}(\widehat{\mathcal{O}})$ with $\gamma \in \mathfrak{a}$ and $\operatorname{Ad}(a) \gamma \in \mathfrak{a}$, then there is $g \in N_{K}(\mathfrak{a})$ such that $g \cdot F=a \cdot F$. Since $\gamma \in \mathfrak{a} \cap$ $\operatorname{Ad}\left(a^{-1}\right) \mathfrak{a}$, both $\mathfrak{a}$ and $\operatorname{Ad}\left(a^{-1}\right) \mathfrak{a}$ are maximal subalgebras in $\mathfrak{p}^{\gamma}$. Hence there is $g \in K^{\gamma}=H_{F}$ such that $\operatorname{Ad}\left(a^{-1}\right) \mathfrak{a}=\operatorname{Ad}(g) \mathfrak{a}$. Therefore $w:=a g \in N_{K}(\mathfrak{a})$ and $a \cdot F=a g \cdot F=w \cdot F$. 
Define a map

$$
\varphi: \mathscr{F}(\widehat{\mathcal{O}}) / K \rightarrow \mathscr{F}(P) / W
$$

by the following rule: given a class in $\mathscr{F}(\widehat{\mathcal{O}}) / K$ choose a representative $F$ such that $\mathfrak{s}_{F} \subset \mathfrak{a}$ and set $\varphi([F]):=[F \cap \mathfrak{a}]$. By Proposition $3.6 F \cap \mathfrak{a}$ is indeed a face of the polytope and by Lemma 3.8 a different choice of the representative will yield the same class in $\mathscr{F}(P) / W$, so that the map $\varphi$ is well-defined.

Now fix a face $F$ with $\mathfrak{s}_{F} \subset \mathfrak{a}$. $F$ is an orbitope of $G_{F}^{0}$. Applying Proposition 3.5 we get a decomposition $\mathfrak{g}_{F}=\mathfrak{g}_{1} \oplus \mathfrak{g}_{2} \oplus \mathfrak{g}_{3}$ like (3.1). Here $\mathfrak{g}_{3}=$ $\mathfrak{z}\left(\mathfrak{g}_{F}\right)$. Accordingly $\mathfrak{a}=\mathfrak{a}_{1} \oplus \mathfrak{a}_{2} \oplus \mathfrak{s}_{F}$, where $\mathfrak{a}_{i}:=\mathfrak{a} \cap \mathfrak{g}_{i}$ is a maximal subalgebra of $\mathfrak{p}_{i}$ for $i=1,2$. We have used the fact that $\mathfrak{p}_{3}=\mathfrak{z}\left(\mathfrak{g}_{F}\right) \cap \mathfrak{p}=\mathfrak{s}_{F}$ by Corollary (3.5). Denote by $W_{1}$ and $W_{2}$ the Weyl groups of $\left(\mathfrak{g}_{1}, \mathfrak{a}_{1}\right)$ and $\left(\mathfrak{g}_{2}, \mathfrak{a}_{2}\right)$. They can be considered as subgroups of $W=W(\mathfrak{g}, \mathfrak{a})$. They commute and have the following sets of invariant vectors:

$$
\mathfrak{a}^{W_{1}}=\mathfrak{a}_{2} \oplus \mathfrak{s}_{F} \quad \mathfrak{a}^{W_{2}}=\mathfrak{a}_{1} \oplus \mathfrak{s}_{F} \quad \mathfrak{a}^{W_{1} \times W_{2}}=\mathfrak{s}_{F}
$$

Lemma 3.9. Let $F \subset \widehat{\mathcal{O}}$ be a non-empty face with $\mathfrak{s}_{F} \subset \mathfrak{a}$. Set $\sigma:=F \cap \mathfrak{a}$. Then $W_{1} \times W_{2}$ preserves $\sigma$.

Proof. Recall from Proposition 3.5 that ext $F=x_{0}+K_{1} \cdot x_{1}$. By Kostant theorem $\quad \sigma=\pi(\operatorname{ext} F)=x_{0}+\operatorname{conv}\left(W_{1} \cdot x_{1}\right)=\operatorname{conv}\left(W_{1} \cdot x\right)$. Hence $W_{1}$ preserves $\sigma$. Moreover $\sigma \subset \mathfrak{s}_{F} \oplus \mathfrak{a}_{1}$ hence $W_{2}$ fixes $\sigma$ pointwise and the statement follows.

If $\sigma$ is a face of $P$ set $G_{\sigma}:=\{g \in W: g(\sigma)=\sigma\}$.

Lemma 3.10. If $\sigma \in \mathscr{F}(P)$ there is a vector $\beta \in \mathfrak{a}$ that is fixed by $G_{\sigma}$ and such that $\sigma=F_{\beta}(P)$. If $\beta$ is any such vector and $F:=F_{\beta}(\widehat{\mathcal{O}})$, then $F \cap \mathfrak{a}=\sigma, G_{\sigma}=W_{1} \times W_{2}, \mathfrak{s}_{F}=\mathfrak{a}^{G_{\sigma}}$ and $F$ depends only on $\sigma$, not on the choice of $\beta$.

Proof. The existence of a $G_{\sigma}$-invariant $\beta$ such that $F_{\beta}(P)=\sigma$ follows directly from Lemma 2.3. If $F:=F_{\beta}(\widehat{\mathcal{O}})$ it follows immediately that $F \cap \mathfrak{a}=\sigma$. By Lemma $3.9 W_{1} \times W_{2} \subset G_{\sigma}$, so $\beta \in \mathfrak{a}^{G_{\sigma}} \subset \mathfrak{a}^{W_{1} \times W_{2}}=\mathfrak{s}_{F}$. It follows that $H_{F}=K^{\beta}$. The subgroup of $W$ that fixes $\beta$ is the Weyl group of $\left(\mathfrak{g}^{\beta}, \mathfrak{a}\right)$, i.e., $W_{1} \times W_{2}$. Hence $W_{1} \times W_{2}=G_{\sigma}$ and $\mathfrak{s}_{F}=\mathfrak{a}^{G_{\sigma}}$. So $\mathfrak{s}_{F}$ depends only on $\sigma$, not on the choice of $\beta$. The same holds for $H_{F}=K^{\mathfrak{s}_{F}}$ and for ext $F$, which is equal to the $H_{F}$-orbit through a point in $\operatorname{ext} \sigma$. 
Define a map $\psi: \mathscr{F}(P) / W \rightarrow \mathscr{F}(\widehat{\mathcal{O}}) / K$ by the following rule: given $\sigma$, fix $\beta \in \mathfrak{a}^{G_{\sigma}}$ such that $\sigma=F_{\beta}(P)$ and set $\psi([\sigma]):=\left[F_{\beta}(\widehat{\mathcal{O}})\right]$. By the previous lemma $F_{\beta}(\widehat{\mathcal{O}})$ depends only on $\sigma$, not on $\beta$. It is clear that $\psi$ is well-defined on equivalence classes.

Theorem 1.1. The maps $\psi$ and $\varphi$ are inverse to each other. Therefore $\mathscr{F}(P) / W$ and $\mathscr{F}(\widehat{\mathcal{O}}) / K$ are in bijective correspondence.

Proof. Let $\sigma$ be a face of $P$. Choose $\beta \in \mathfrak{a}^{G_{\sigma}}$ such that $\sigma=F_{\beta}(P)$. If $F:=$ $F_{\beta}(\widehat{\mathcal{O}})$, then $\mathfrak{s}_{F} \subset \mathfrak{a}$. So $\varphi \circ \psi([\sigma])=\varphi([F])=[F \cap \mathfrak{a}]=[\sigma]$ and $\varphi \circ \psi$ is the identity. Thus $\varphi$ is surjective. It is enough to show that $\varphi$ is injective. Let $F_{1}, F_{2} \subset \widehat{\mathcal{O}}$ be faces such that $\varphi\left(\left[F_{1}\right]\right)=\varphi\left(\left[F_{2}\right]\right)$. Acting with $K$ we can assume that both $\mathfrak{s}_{F_{1}}$ and $\mathfrak{s}_{F_{2}}$ are contained in $\mathfrak{a}$. Acting with $W$ we can also assume that $F_{1} \cap \mathfrak{a}=F_{2} \cap \mathfrak{a}$. By Corollary 3.2 , we get $F_{1}=F_{2}$. By Proposition 3.6 the map between $\mathscr{F}(P) / W$ and $\mathscr{F}(\widehat{\mathcal{O}}) / K$ is the one stated in the introduction.

Remark 3.1. Let $K_{1} \rightarrow \mathrm{O}(V)$ be a polar representation. By Dadok's theorem there is a semisimple Lie group $G$ with Cartan decomposition $\mathfrak{g}=\mathfrak{k} \oplus \mathfrak{p}$ such that $V=\mathfrak{p}$ and the orbits of $K_{1}$ coincide with the orbit of $\operatorname{Ad} K$. A maximal subalgebra $\mathfrak{a} \subset \mathfrak{p}$ is a section for both actions. Denote by $W$ the Weyl group of $(\mathfrak{g}, \mathfrak{a})$ and by $W_{1}$ the Weyl group of the polar representation of $K_{1}$. If $x \in \mathfrak{a}$, then $W \cdot x=K \cdot x \cap \mathfrak{a}=K_{1} \cdot x \cap \mathfrak{a}=W_{1} \cdot x$. We claim that $\mathscr{F}(\widehat{\mathcal{O}}) / K_{1}=\mathscr{F}(\widehat{\mathcal{O}}) / K$ and $\mathscr{F}(P) / W_{1}=\mathscr{F}(P) / W$. Indeed let $F \in \mathscr{F}(\widehat{\mathcal{O}})$ and $k \in K$. Fix a point $x \in \operatorname{relint} F$. There is some $k_{1} \in K_{1}$ such that $k_{1} x=k x$. Then $k x$ belongs both to relint $k F$ and to relint $k_{1} F$. Hence $k F=k_{1} F$ by Theorem 2.1. This shows that the $K$-orbit through $F$ is contained in the $K_{1}$-orbit through $F$. Interchanging $K$ and $K_{1}$ we get the opposite inclusion. Thus $\mathscr{F}(\widehat{\mathcal{O}}) / K_{1}=\mathscr{F}(\widehat{\mathcal{O}}) / K$. In the same way one proves that $\mathscr{F}(P) / W_{1}=\mathscr{F}(P) / W$. From this it follows that Theorem 1.1 holds for any polar representation.

\section{Final remarks}

It follows from the results in the previous section that there are a finite number of $K$-orbits on the set $\mathscr{F}(\widehat{\mathcal{O}})$. Given such an orbit, we denote by $S$ the union of the faces in the orbit. Therefore $S$ equals $K \cdot F$ for some face $F \in \mathscr{F}(\widehat{\mathcal{O}})$. We call $S$ the stratum corresponding to the face $F$. Arguing as in the case of coadjoint orbitopes [2, Section 5] one proves the following. 
Theorem 4.1. The strata give a partition of $\partial \widehat{\mathcal{O}}$. They are smooth embedded submanifolds of $\mathfrak{p}$ and are locally closed in $\partial \widehat{\mathcal{O}}$. For any stratum $S$ the boundary $\bar{S}-S$ is the disjoint union of strata of lower dimension.

The computation of the dimension of the strata is trickier in this case. Nevertheless the bound in the statement follows easily from the following argument. If $E$ is an $n$-dimensional convex body, then $\partial E$ has Hausdorff dimension $n-1$. If $F$ is an $n$-dimensional face, the boundary of the stratum $S:=K \cdot F$ is a fiber bundle over a compact base with fibers isometric to $\partial F$. Therefore, its Hausdorff dimension is strictly smaller than the dimension of $S$.

Also the description of the faces of $\widehat{\mathcal{O}}$ and of the momentum polytope in terms of root data is just as in the case of coadjoint orbitopes (see Section 6 in [2]). We briefly state the result.

Fix a maximal subalgebra $\mathfrak{a}$ of $\mathfrak{p}$ and a system of simple roots $\Pi \subset \Delta=$ $\Delta(\mathfrak{g}, \mathfrak{a})$. A subset $E \subset \mathfrak{a}$ is connected if there is no pair of disjoint subsets $D, C \subset E$ such that $D \sqcup C=E$, and $\langle x, y\rangle=0$ for any $x \in D$ and for any $y \in C$. (A thorough discussion of connected subsets can be found in [23], [20, Section 5].) Connected components are defined as usual. If $x$ is a non-zero vector of $\mathfrak{a}$, a subset $I \subset \Pi$ is called $x$-connected if $I \cup\{x\}$ is connected. Equivalently, $I \subset \Pi$ is $x$-connected if and only if every connected component of $I$ contains at least one root $\alpha$ such that $\alpha(x) \neq 0$. If $I \subset \Pi$ is $x$-connected, denote by $I^{\prime}$ the collection of all simple roots orthogonal to $\{x\} \cup I$. The set $J:=I \cup I^{\prime}$ is called the $x$-saturation of $I$. The largest $x$-connected subset contained in $J$ is $I$. So $J$ is determined by $I$ and $I$ is determined by $J$. Given a subset $I \subset \Pi$ we will denote by $Q_{I}$ the parabolic subgroup with Lie algebra $\mathfrak{q}_{I}$ as defined in (2.2).

Theorem 4.2. Let $\mathcal{O} \subset \mathfrak{p}$ be a $K$-orbit and let $x$ be the unique point in $\mathcal{O} \cap \bar{C}$.

(a) If $I \subset \Pi$ is $x$-connected and $J$ is its $x$-saturation, then $Q_{I} \cdot x=Q_{J} \cdot x$ and $F:=\operatorname{conv}\left(Q_{J} \cdot x\right)$ is a face of $\widehat{\mathcal{O}}$. If $\beta \in \mathfrak{a}_{J}$ and $\lambda(\beta)>0$ for any $\lambda \in \Pi-J$, then $F=F_{\beta}(\widehat{\mathcal{O}})$. Moreover $Q_{F}=Q_{J}$.

(b) Any face of $\widehat{\mathcal{O}}$ is conjugate to one of the faces constructed in (a).

\section{Acknowledgments}

The first two authors are grateful to the Fakultät für Mathematik of RuhrUniversität Bochum for the wonderful hospitality. We also would like to 
thank the referees for helpful comments. The first and second authors were partially supported by GNSAGA of INdAM and by FIRB 2012 "Geometria differenziale e teoria geometrica delle funzioni". The second author was also supported by PRIN 2009 MIUR "Moduli, strutture geometriche e loro applicazioni". The third author was partially supported by DFG-priority program SPP 1388 (Darstellungstheorie).

\section{References}

[1] L. Biliotti and A. Ghigi, Satake-Furstenberg compactifications, the moment map and $\lambda_{1}$, Amer. J. Math. 135(1) (2013), 237-274.

[2] L. Biliotti, A. Ghigi and P. Heinzner, Coadjoint orbitopes, arXiv:math.RT/1110.6039, 2011, to appear on Osaka J. Math.

[3] A. Borel and L. Ji, Compactifications of symmetric and locally symmetric spaces, Mathematics: Theory \& Applications. Birkhäuser Boston Inc., Boston, MA, 2006.

[4] J.-L. Clerc and K.-H. Neeb, Orbits of triples in the Shilov boundary of a bounded symmetric domain, Transform. Groups, 11(3) (2006), 387-426.

[5] J. Dadok, Polar coordinates induced by actions of compact Lie groups, Trans. Amer. Math. Soc. 288(1) (1985), 125-137.

[6] J.J. Duistermaat, J.A.C. Kolk and V.S. Varadarajan, Functions, flows and oscillatory integrals on flag manifolds and conjugacy classes in real semisimple Lie groups, Compos. Math., 49(3) (1983), 309-398.

[7] V.M. Gichev, Polar representations of compact groups and convex hulls of their orbits, Differ. Geom. Appl. 28(5) (2010), 608-614.

[8] G. Heckman, Projection of orbits and asymptotic behaviour of multiplicities of compact Lie groups, 1980, $\mathrm{PhD}$ thesis.

[9] P. Heinzner and P. Schützdeller, Convexity properties of gradient maps, Adv. Math. 225(3) (2010), 1119-1133.

[10] P. Heinzner, G.W. Schwarz and H. Stötzel, Stratifications with respect to actions of real reductive groups, Compos. Math. 144(1) (2008), 163-185. 
[11] P. Heinzner and H. Stötzel, Critical points of the square of the momentum map, In Global aspects of complex geometry, 211-226, Springer, Berlin, 2006.

[12] P. Heinzner and H. Stötzel, Semistable points with respect to real forms, Math. Ann. 338(1) (2007), 1-9.

[13] S. Helgason. Differential geometry, Lie groups, and symmetric spaces, volume 80 of Pure and Applied Mathematics, Academic Press Inc., New York, 1978.

[14] J.E. Humphreys, Introduction to Lie algebras and representation theory, Graduate Texts in Mathematics 9, Springer-Verlag, New York, 1978, Second printing, revised.

[15] A.A. Kirillov, Lectures on the orbit method, volume 64 of Graduate Studies in Mathematics, American Mathematical Society, Providence, RI, 2004.

[16] A.W. Knapp, Lie groups beyond an introduction, Volume 140 of Progress in Mathematics, Birkhäuser Boston Inc., Boston, MA, 2nd edition, 2002.

[17] A. Korányi, Remarks on the Satake compactifications, Pure Appl. Math. Q. 1(4, part 3) (2005), 851-866.

[18] B. Kostant, On convexity, the Weyl group and the Iwasawa decomposition, Ann. Sci. École Norm. Sup. (4) 6 413-455, (1974), 1973.

[19] C. Miebach, Geometry of invariant subsets in complex semi-simple Lie groups, Dissertation, Ruhr-Universität Bochum, 2007.

[20] C.C. Moore, Compactifications of symmetric spaces, Amer. J. Math. 86 (1964), 201-218.

[21] R.S. Palais and C.-L. Terng, Critical point theory and submanifold geometry, volume 1353 of Lecture Notes in Mathematics. SpringerVerlag, Berlin, 1988.

[22] R. Sanyal, F. Sottile and B. Sturmfels, Orbitopes, Mathematika 57 (2011), 275-314.

[23] I. Satake, On representations and compactifications of symmetric Riemannian spaces, Ann. Math. (2) 71 (1960), 77-110. 
[24] R. Schneider, Convex bodies: the Brunn-Minkowski theory, Volume 44 of Encyclopedia of Mathematics and its Applications, Cambridge University Press, Cambridge, 1993.

[25] C.-L. Terng, Convexity theorem for isoparametric submanifolds, Invent. Math. 85(3) (1986), 487-492.

Dipartimento di Matematica

UNIVERSITA DI PARMA

Viale G. Usberti 53/A

43100 PARMA

E-mail address: leonardo.biliotti@unipr.it

Dipartimento di Matematica e Applicazioni

Università di Milano BicocCA

VIA COZZI 53

20125 Milano

ITALIA

E-mail address: alessandro.ghigi@unimib.it

RUHR UNIVERSITÄT BOCHUM

FAKUltat FÄr MATHEMATIK

RAUM NA 4/74

D-44780 BoChum

E-mail address: peter.heinzner@rub.de

Received June 25, 2012 